\title{
UNE ÉTUDE SUR LES COURBES DE LACTATION
}

\author{
PAR
}

\author{
J. DELAGE, A. M. LEROY, J. POLY
}

Laboratoire de Recherches Zootechniques de 1'Institut National Agronomique

\section{PLAN DU MEMOIRE}

\section{I. - Introduction. Les caractéristiques d'une lactation. \\ II. - Les courbes de lactation. \\ I. - Les travaux antérieurs :}

A) Phase ascendante de la lactation.

B) Phase de décroissance de la lactation.

a) Expressions mathématiques.

b) Expressions numériques.

c) Expressions graphiques.

d) Traite du matin et du soir.

C) Discussion.

\section{2. - Etude expérimentale:}

A) Matériel d'étude.

B) Méthode utilisée.

C) Étude de la courbe moyenne :

a) Phase ascendante de la courbe de lactation.

b) Phase descendante de la courbe de lactation.

Étude de la courbe de lactation

moyenne du $60^{e}$ jour au $25^{\circ}$ jour après le vêlage :

Hypothèse linéaire.

Hypothèse exponentielle.

Hypothèse hyperbolique.

Hypothèse paraboliques.

Interprétation de la courbe de lactation entre $1 \mathrm{e} 6 \mathrm{o}^{\mathrm{e}}$ et le $25^{\mathrm{e}}$ jour ; conclusions.

Étude de la courbe de lactation moyenne du $25^{\mathrm{e}}$ jour au $300^{\mathrm{e}}$ jour.

c) Effet de la gestation sur la lactation :

- travaux antérieurs,

- nos résultats.

d) Conclusions de l'étude de la courbe moyenne.

III. - Conclusion.

IV. - Résumé.

Bibliographie. 


\section{I. - INTRODUCTION}

\section{LES CARACTÉRISTIQUES D'UNE LACTATION}

La production du lait évolue au cours d'une lactation suivant un cycle qui est de même nature chez toutes les vaches laitières. La production journalière s'accroît pendant les premières semaines qui suivent le vêlage, passe par un maximum à une date variable selon les animaux, puis diminue pluss ou moins régulièrement jusqu'au tarissement. Cette évolution de la production peut se représenter graphiquement par une courbe de lactation - (schématisée sur la fig. I) - obtenue en portant en abscisses le temps écoulé depuis le vêlage, et en ordonnées les productions journalières correspondantes, exprimées en $\mathrm{kg}$ de lait réellement fournis, ou en leur équivalent énergétique, en kg de lait à $4 \%$ (r).

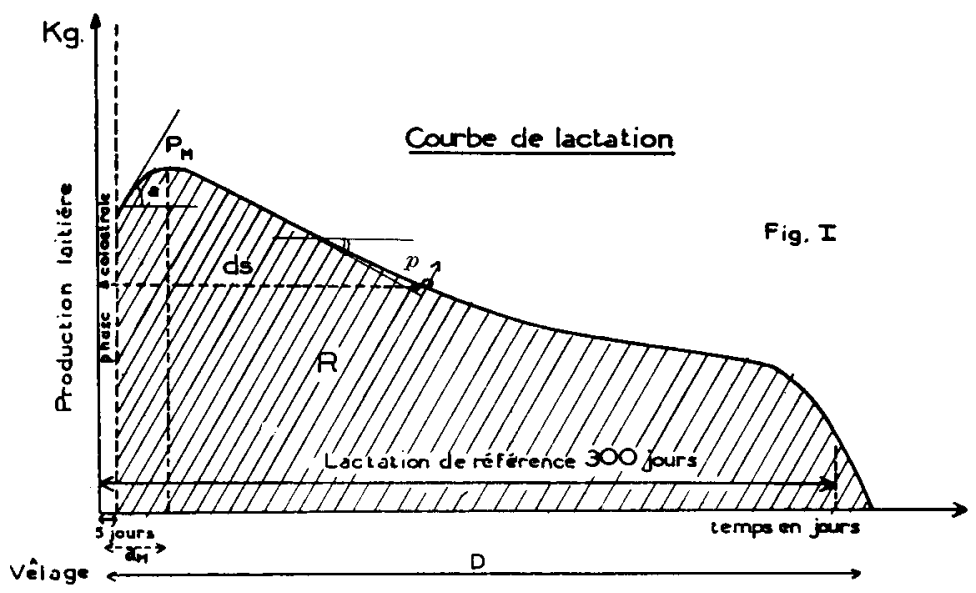

Des variations individuelles importantes s'observent dans les formes des courbes de lactation; il est bien connu que deux animaux peuvent fournir une même production totale au cours de leur lactation en répartissant différemment leurs productions journalières, ainsi qu'en témoignent les courbes observées par EsKEDAL au Danemark $(2,3)$ et par nous-mêmes en Seine-et-Marne. I,es courbes de la figure 2, empruntées aux archives du Syndicat d'Elevage de Seine-et-Marne, correspondent à des vaches $\mathrm{A}$ et $\mathrm{B}$ de même âge, qui ont vêlé en mars 1949 et ont donné 4 ooo $\mathrm{kg}$ de lait environ, en 300 jours ; l'allure des courbes A et B est pourtant très différente. De nombreux cas similaires pourraient être cités.

I a production totale d'une vache au cours d'une lactation ne fournit donc qu'une appréciation incomplète de cette lactation et l'évolution des productions journalières ne saurait être négligée. I1 importe alors de 
chercher à caractériser systématiquement les courbes de lactation. A cette fin, 6 paramètres peuvent être retenus (fig. I) :

- la production totale, $\mathrm{R}$;

- le rythme de croissance de la production dans la phase ascendante de la lactation, du vêlage à la production maximum, qu'on pourrait défi-

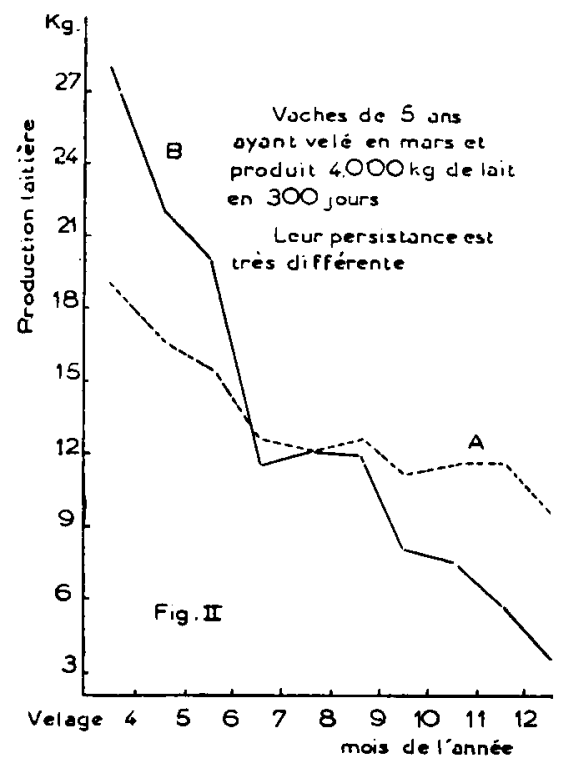

nir graphiquement en tout point de cette période par le paramètre angulaire, $a$;

- la production journalière maximum, $\mathrm{P}_{\mathrm{M}}$;

- la date à laquelle commence la décroissance de la production, $d_{\mathrm{M}}$;

- la persistance de la lactation, qui traduit le taux de décroissance dans la $2^{\text {e }}$ phase de la lactation et qui, en tout point de cette période, pourrait s'exprimer graphiquement par le paramètre angulaire, $p$;

- la durée de la lactation, D.

\section{II. - LES COURBES DE LACTATION \\ I. - LES TRAVAUX ANTERIEURS}

\section{A) Phase ascendante de la lactation}

Par suite de l'insuffisance des données mensuelles ou même bimensuelles de contrôle laitier, peu d'études très approfondies ont été effectuées sur la phase ascendante de la lactation; HAEcker (4) rapporte déjà en I903, dans une étude portant sur 239 animaux, que la meilleure 
semaine de la lactation est la $3^{\mathrm{e}}$ semaine. TURNER et coll. (5) travaillant avec 80 Holstein-Firiesian contrôlées journellement pendant les 90 premiers jours de la lactation, constatent que la production est maximum vers les $15^{\mathrm{e}}$ et $\mathrm{I} 6^{\mathrm{e}}$ jours après le vêlage pour les vaches traites deux fois, vers le $\mathrm{I} 8 \mathrm{e}$ jour pour celles qui sont traites trois fois et vers le $28^{\mathbf{e}}$ jour pour les 8 animaux qui le sont quatre fois. Ils en concluent alors que la fréquence des traites et la quantité de lait produite sont les facteurs les plus importants qui régissent l'époque du maximum de production. Il faut en effet plus de temps, selon les auteurs, pour adapter à des besoins élevés de production un haut niveau de nutrition. Mc CANDLISH (6) travaillant avec le troupeau de l'état d'Iowa montre que chez les vaches Holstein et Ayrshire le maximum de production est atteint dans le $2^{\mathrm{e}}$ mois de lactation, alors que Jersey et Guernesey réalisent ce maximum dans

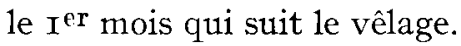

DRAKELEY et coll. (7), en Angleterre, constatent par contre que les vaches Guernesey, British Friesian, Dairy Shorthorn atteignent leur pointe de production vers le $30^{\mathbf{e}}$ jour après le vêlage, les Ayrshire vers le $35^{\mathrm{e}}$ jour et les Jersey vers le $45^{\mathrm{e}}$ jour. Gavin (8), sur un ensemble de I 42 I performances qu'il a étudiées, trouve que $84 \%$ des vaches atteignent leur maximum dans les 8 semaines après le vêlage, $92 \%$ dans les I 2 semaines, $97 \%$ dans les $\mathbf{1} 6$ semaines.

Pour des animaux Shorthorn, BARTLETT (9) constate un maximum de production vers le $50^{\mathrm{e}}$ jour et OxiEy (ro) vers le $30^{\mathrm{e}}$ jour.

OSTERGAARD (II), au cours de recherches statistiques sur la production laitière de vaches danoises inscrites au Herd-Book, situe le maximum de la lactation entre les $30^{\mathrm{e}}$ et $40^{\mathrm{e}}$ jours qui suivent le vêlage.

MöLLGAARD (I2) admet une phase de croissance dans la lactation avec un maximum entre la $4^{\mathrm{e}}$ et la $8^{\mathrm{e}}$ semaine, suivie d'une phase stationnaire avant la période de décroissance de la production.

Pour LEROY et coll. (I3), les plus hautes performances journalières se situent dans le $2^{\mathrm{e}}$ mois suivant le vêlage.

E.dWARds (I4), dans une étude récente portant sur quelque 2 ooo animaux trouve que le maximum de production est dépassé après le $45^{\mathrm{e}}$ jour.

Enfin, d'après Csukas (I5), la phase ascendante plafonne entre la Ire et la ${ }^{2}{ }^{e}$ semaine de lactation, avec d'importantes variations individuelles, par conséquent. Il conclut d'ailleurs, que cette phase est caractéristique d'un animal donné et paraît conditionnée essentiellement par des facteurs héréditaires.

\section{B) Phase de décroissance}

La phase de décroissance de la lactation, étudiée pour la première fois par STURTEVANT (I6) en r886, a surtout retenu l'attention des divers 
auteurs. Ils ont utilisé des modes d'expression différents pour la caractériser, souvent, d'ailleurs parce qu'était différent le but des études qu'ils avaient entreprises.

Les divers modes d'expression peuvent être classés en 3 groupes :

a) les expressions mathématiques,

b) les expressions numériques diverses exprimées sous forme de rapports entre des productions obtenues à des stades différents de la lactation,

c) les expressions graphiques.

\section{a) Les expressions mathématiques}

Peu d'études systématiques ont été entreprises en vue de rechercher la meilleure expression mathématique qui permette de caractériser une courbe de lactation. Par contre, certains auteurs ont proposé de définir, en tout ou en partie, les courbes par une fonction déterminée. Deux types ont été suggérés : 1a droite, l'exponentielle.

\section{LA DROITE :}

GAINES (I7) passant en revue les divers modes d'expression de la persistance de la lactation, suggère de représenter la décroissance de la production par l'équation linéaire :

$$
y=\mathrm{C}-\mathrm{B} t,
$$

dans laquelle $y$ représente la production; $\mathrm{C}$, la production initiale; $t$, le temps écoulé depuis le vêlage. La constante B est une mesure absolue de la persistance.

Il a d'ailleurs rejeté ce mode de représentation pour adopter une équation exponentielle.

BonnIer (I8) en I935, se limitant à l'étude des I3 premières semaines qui suivent la production maximum, interprète linéairement la décroissance de la production de lait à $4 \%$, durant cette période. Il caractérise cette décroissance par le coefficient de régression linéaire correspondant. Ses travaux, qui avaient pour but la comparaison de portions semblables de courbes chez des mères et des filles, ne permettent pas d'en généraliser les résultats à l'ensemble de la courbe de lactation.

KRONACHER et coll. (I9), divisant la courbe de lactation en 3 parties, adaptent également la phase intermédiaire de décroissance comprise entre la production maximum et l'époque où l'effet de la gestation devient sensible, à une régression linéaire.

\section{L'EXPONENTIELLE:}

La majorité des auteurs a admis implicitement à la suite des travaux Annales de Zootechnie. - 1953 . 
de Brody, Ragsdale, Turner $(5,20,2 \mathrm{I})$ que la décroissance de la production laitière s'effectuait selon une loi exponentielle,

$$
\mathrm{M}_{t}=\mathrm{M}_{0} e^{-k t}
$$

$t$, représentant le temps écoulé depuis le vêlage, $\mathrm{M}_{t}$ la production laitière au temps $t, \mathrm{M}_{0}$ la production laitière théorique au vêlage, et $k$ étant une constante.

Cette loi de décroissance exponentielle peut s'exprimer sous une forme plus pratique, comme le font remarquer les auteurs ; en effet, cela revient à dire que la production de chaque mois est un pourcentage constant de la production du mois précédent. Ainsi, avec leurs données, les auteurs calculaient que la production mensuelle de vaches Guernesey représentait $94 \%$ de la production du mois précédent.

Gaines ( $17,22,23$ ), Gaines et Davidson (24) admettent également une décroissance exponentielle de la production laitière. GaINEs (25) donne une interprétation intéressante du cycle de production d'une vache laitière. La surface hachurée sur la figure 3 correspond à l'intégrale

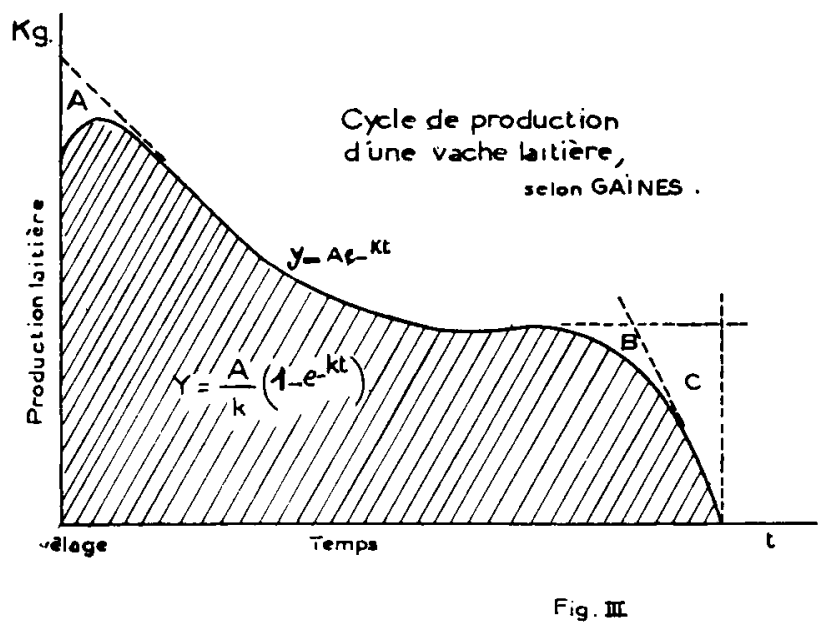

de la courbe qui la limite et à la production totale fournie par l'animal au cours de sa lactation. La surface A correspond à une perte de production due au fait que l'animal n'a pas sa production maximum au vêlage, mais qu'il lui faut un certain temps pour réaliser sa pleine capacité de production ; l'auteur interprète ce retard comme une action résiduelle de la gestation antérieure. I $\mathrm{L}$ surface $\mathrm{B}$ correspond à une perte de production imputable à l'effet sensible de la gestation, un certain temps après la saillie. Quant à la surface C, elle correspond aux effets plus ou moins artificiels du tarissement.

La production totale fournie par l'animal au temps $t$ est alors:

$$
\mathrm{Y}=\frac{\mathrm{A}}{\mathrm{K}}\left(\mathrm{I}-e^{-\mathrm{R} t}\right) \text {. }
$$


Garnes (I7) étudiant les modes d'expression de la persistance conclut que la constante $\mathbf{K}$ de l'équation exponentielle est la seule vraie mesure mathématique de la persistance; $K$ traduit en effet le rythme de changement mensuel dans la production; mais la constante $\mathrm{K}$ est ainsi inversement proportionnelle à la persistance.

Gooch (26) utilise également une exponentielle de la forme $y=\mathrm{A} e^{b t}$ pour caractériser la décroissance de la production dans les 8 premiers mois suivant le vêlage pour 679 lactations de Jersey; $e^{b}$ fournit une mesure de la persistance de la lactation, qui lui est directement proportionnelle.

PonTECORvo (27) dans une étude génétique sur la persistance dans un troupeau de vaches Ayrshire, caractérise la courbe de lactation comprise entre le $50^{\mathrm{e}}$ et le $240^{\mathrm{e}}$ jour par une loi exponentielle : $y=\mathrm{A} e^{-k t}$.

Il se limite à cette période après avoir distingué dans la courbe de lactation totale les 3 phases suivantes :

a) Une phase ascendante après le vêlage correspondant aux 50 premiers jours ;

b) Une phase de décroissance non perturbée par la gestation entre le $50^{\mathrm{e}}$ et le $240^{\mathrm{e}}$ jour ;

c) Une phase terminale à décroissance rapide après le $240^{\mathrm{e}}$ jour, au début de laquelle l'influence de la gestation apparaît.

Dans la phase intermédiaire, PONTECoRvo caractérise la décroissance par un coefficient de persistance:

$$
\mathrm{P}=\mathrm{IOO} \cdot e^{-k}
$$

dont la valeur moyenne, pour I63 lactations, est de : 88,42 $\pm 0,36$. Le pourcentage constant de diminution mensuelle de la production est donc de $I I, 58 \%$.

EDWARDS (I4) trouve en coordonnées semi-logarithmiques, une décroissance linéaire de la production, ce qui confirme la nature exponentielle de la $2^{\Theta}$ phase de la lactation.

SrkKA (28) utilisant les données de la "Scottish Milk Recording Association " préfère — pour 48 lactations sur Ioo qu'il a étudiées — à l'expression exponentielle classique représentant la phase de décroissance de la lactation, une expression exponentielle parabolique de la forme:

$$
y=\mathrm{A} e^{b t+c t^{2}}
$$

dans laquelle la constante linéaire $b$ traduit le rythme moyen de diminution de la production, et dans laquelle la constante parabolique $c$ exprime la variation de ce rythme, au cours de périodes successives. 


\section{b) Expressions diverses}

Elles ont toutes pour principe, de caractériser la persistance de la lactation par des rapports entre des productions obtenues au cours de diverses périodes.

a) RAPPORT DE LA PRODUCTION D'UN MOIS A CELIE DU MOIS PRÉCÉDENT :

Sturtevant (i6), Linfield (29), Grady (30), Turner (3I) caractérisent la décroissance de la production en exprimant chaque production mensuelle en pourcentage de la production du mois précédent. STURTEVANT et LINFIELD constatent avec leurs animaux une baisse mensuelle de production de $9 \%$. GRADY distingue des vaches qui sont persistantes et qui, au cours des 6 premiers mois de la lactation présentent une baisse mensuelle de la production de 1'ordre de $3 \%$, d'autres qui ne le sont pas, avec une chute mensuelle de io \%. Pour Turner, qui admettait une décroissance exponentielle de la production laitière, la persistance est donnée par la moyenne des pourcentages mensuels de persistance.

L'un de nous préconisait plus tard l'emploi de cette méthode pour contrôler les conditions d'affourragement dans une étable ( $13,32,33)$. En désignant par $\mathrm{M}$ la production moyenne observée pendant le $2^{\mathbf{e}}$ mois qui suit le vêlage, et par $A_{1}, A_{2}, A_{3} \ldots . . A n-I, A n$, les productions quotidiennes moyennes des premier, deuxième, ...nième mois, qui suivent ce mois considéré comme mois de production maximum, on observe ordinairement la série d'égalités suivantes :

$$
\frac{\mathrm{A}_{1}}{\mathrm{M}}=\frac{\mathrm{A}_{2}}{\mathrm{~A}_{1}}=\frac{\mathrm{A}_{3}}{\mathrm{~A}_{2}}=\cdots \frac{\mathrm{A}_{n}}{\mathrm{~A}_{(n-1)}}=\mathrm{K} .
$$

Dans la pratique, $\mathrm{K}$ se calcule en faisant la moyenne arithmétique de ces différents rapports et $K$. Ioo est le coefficient de persistance de la lactation.

BÜNGER (34) effectuant en Allemagne des études similaires, caractérisait également 1'évolution des lactations de son troupeau expérimental, contrôlé hebdomadairement, en exprimant chaque production mensuelle en pourcentage de la production du mois précédent.

ङ) RAPPORT DE LA PRODUC'TION TOTALE A LA PRODUCTION MAXIMUM:

$$
\text { "shape-figure" de SANDERS: }
$$

Au cours de nombreux et importants travaux sur les diverses influences externes agissant sur la production laitière et d'études plus particulièrement centrées sur la courbe de lactation $(35,36)$ SANDERS définit un indice S. F (shape-figure) comme le rapport entre la production totale $\mathrm{P}$ 
et la production hebdomadaire maximum, rapport corrigé en fonction du mois de vêlage par un coefficient $R$.

$$
\text { S. F. }=\frac{\mathrm{P}}{\mathrm{M}} \cdot \frac{\mathrm{I}}{\mathrm{R}} \text {. }
$$

$\mathrm{R}$ est donné par un tableau établi par l'auteur pour les vaches étudiées.

\section{Indice de persistance de TURNER :}

TURNER (3I) a proposé un paramètre analogue, défini comme le rapport de la production totale à la production maximum.

\section{Indice $G$. de FREDERICKEN et OSTERGAARD :}

Ces auteurs (37) expriment la production journalière moyenne en pourcentage de la production maximum enregistrée au cours de la lactation :

$$
\mathrm{G}=\mathrm{IO0} \cdot \frac{a}{a_{1}}
$$

$a$ est la production journalière moyenne, $a_{1}$ la production maximum; pour 6989 lactations, les auteurs trouvent une valeur moyenne de G égale à 70 .

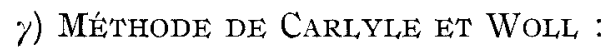

CARLYLE et WOLL (38) utilisent le rapport roo $\frac{a-b}{a}$ dans lequel $a$ et $b$ correspondent à des productions enregistrées au cours de la $3^{\mathrm{e}}$ et de la $\mathbf{r} 3^{\mathrm{e}}$ semaine de la lactation.

ঠ) MÉTHOde DE BRUNN :

BRUUN (39), pour caractériser la persistance d'un animal, utilise le coefficient de variation des diverses productions mensuelles en pourcentage de la production annuelle.

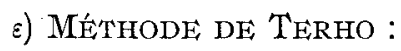

Terho (49), cité par Johansson et HANsson, calcule la production durant les 5 premiers mois après le vêlage en pourcentage de la production totale.

६) Méthodes de Johansson et Johansson et Hansson :

JoHANSSON (4I), s'intéressant à la quantité totale de matières grasses, calcule d'abord la production durant les roo premiers jours de la lactation en pourcentage de la production en 300 jours. 
Plus tard, Johansson et Hansson (42) divisent la période de lactation en 3 périodes de Ioo jours et caractérisent la persistance de la quantité de matières grasses produite, par les pourcentages des productions durant 1a $2^{\mathrm{e}}$ et la $3^{\mathrm{e}}$ période, par rapport à la production durant la I $^{\mathrm{re}}$ période ; si $a, b, c$ représentent les productions durant les $\mathrm{I}^{\mathrm{re}}, 2^{\mathrm{e}}$ et $3^{\mathrm{e}}$ période, ils calculent les pourcentages suivants :

$$
\mathrm{P}_{(2 \cdot 1)}=\frac{100 b}{a} ; \quad \mathrm{P}_{(3 \cdot 1)}=\frac{100 c}{a} .
$$

n) Méthode de Ludwick et Petersen :

Ludwick et PETERSEN (43) distinguent dans une lactation, à partir de la production maximum au $48^{\mathrm{e}}$ jour, 4 divisions physiologiques de 80 jours. L'influence de la gestation apparaîtrait au cours de la $3^{\mathrm{e}}$ période comprise entre le $208^{\mathrm{e}}$ et le $288^{\mathrm{e}}$ jour, chez des vaches qui n'étaient pas encore saillies après 4 mois $\mathbf{I} / 2$ de lactation.

La décroissance de la production est exprimée par les rapports $\frac{X_{2}}{X_{1}}$, $\frac{\mathrm{X}_{3}}{\mathrm{X}_{2}}$ et $\frac{\mathrm{X}_{4}}{\mathrm{X}_{3}}$ dans lesquels $\mathrm{X}_{1}, \mathrm{X}_{2}, \mathrm{X}_{3}, \mathrm{X}_{4}$ représentent re spectivement les productions pour les 4 périodes.

Pour tenir compte de l'importance relative des différents rapports, qui ne sont pas également influencés par le milieu, notamment par la gestation, PeTERSEN les affecte de coefficients propres, et caractérise la persistance par l'expression :

$$
P=\frac{4}{9} \frac{X_{2}}{X_{1}}+\frac{3}{9} \frac{X_{3}}{X_{2}}+\frac{2}{9} \frac{X_{4}}{X_{3}}
$$

I1 souligne d'ailleurs la possibilité d'accroître le nombre de divisions de la période de lactation, et d'utiliser d'autres coefficients de correction, suivant les conditions sous lesquelles sont produites les lactations. $\mathrm{L}_{\mathbf{a}}$ persistance s'exprime alors par la formule générale :

$$
\mathrm{P}=\frac{\frac{\mathrm{X}_{2}}{\mathrm{X}_{1}} \cdot n+\frac{\mathrm{X}_{3}}{\mathrm{X}_{2}} \cdot(n-\mathrm{I})+\cdots+\frac{\mathrm{X}_{n}}{\mathrm{X}_{n-1}}[n-(n-2)]}{n(n-\mathrm{I})-(n-\mathrm{I}) \frac{n-2}{2}},
$$

dans laquelle $n$ représente le nombre de périodes, arbitrairement choisies, pour diviser la lactation. Le dénominateur correspond à la somme des différents coefficients appliqués à chaque rapport du numérateur.

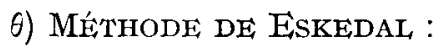

EskEDAI (2), travaillant avec des lactations de 330 jours, les divise en 3 périodes de iro jours, calcule les productions dnrant chacune d'elles et les décroissances de production de période à période. 
i) MÉThode DE MaHadevan :

Enfin, dans une récente et importante étude sur l'effet du milieu et de l'hérédité sur la lactation, MAHADEVAN (44), pour définir la persistance, crée une nouvelle unité de mesure :

$$
\text { Persistance }=\frac{A-B}{B} \text {. }
$$

A est la production laitière pendant les 180 premiers jours de la lactation, et $\mathrm{B}$ la production laitière pendant les Io premières semaines de la lactation.

\section{c) Expressions graphiques}

Tracé D'UNE COURBE DE LACTATION :

Les Syndicats de Contrôle Laitier consignent, en France, les résultats obtenus mensuellement par leurs agents, sur des documents où figurent des courbes de lactation théoriques (fig. 4). Ces courbes, qui résultent des

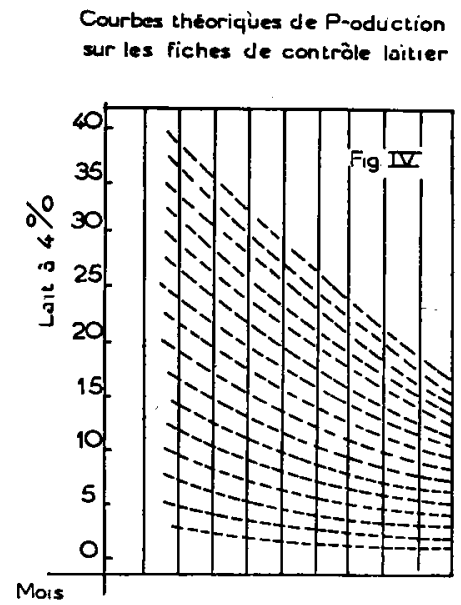

travaux de BRODY et coll., GAINES et coll. IEROY, correspondent à une diminution mensuelle constante de Io \% de la production de lait à $4 \%$ de matières grasses. I a comparaison de la courbe réelle, établie d'après les données des différents contrôles mensuels, avec les courbes théoriques, traduit la plus ou moins grande persistance de la lactation et 1'influence perturbatrice des divers facteurs du milieu, notamment de certaines conditions défavorables d'alimentation.

\section{INDICE P DE NIELSEN :}

NIELSEN (45) cherche à exprimer graphiquement, et à caractériser par un indice, la rapidité de la décroissance de la production qu'il suppose 
linéaire. A cette fin, il construit sur un abaque, à partir d'une origine 0 , une série de Io droites de pentes o à $-\infty$, affectées d'un indice arbitraire $P$, variant de o ̀̀ - Io (sur la figure 5 , les droites de pentes 0 , - I et $-\infty$ sont représentées avec leurs indices correspondants $0,-5,-$ ro).

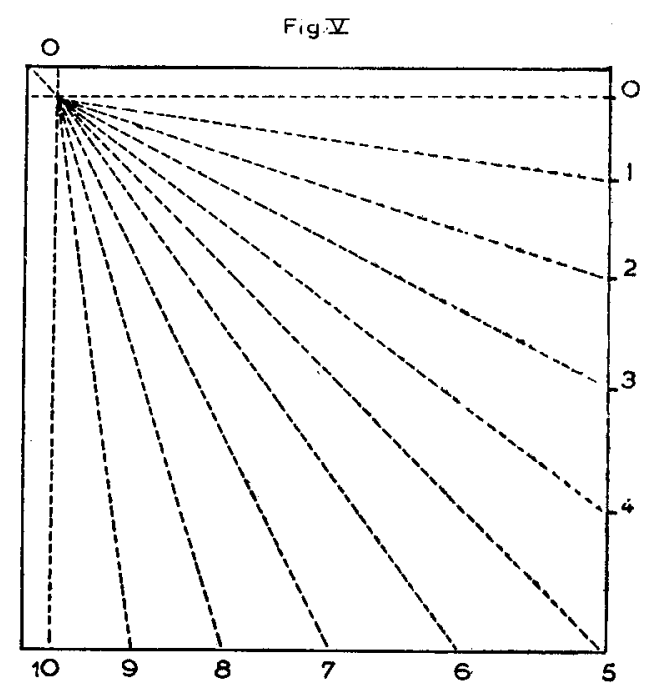

Indice $P$ de Nielsen

NIELSEN définit chaque courbe de lactation par 1'indice de la droite, qui traduit dans les meilleures conditions la décroissance au cours des I3 premières semaines qui suivent la production maximum. Deux courbes $A$ et $B$ représentées sur la figure 6 fournissent des exemples de ce mode de représentation graphique. Une corrélation positive significative existe entre les indices $\mathrm{P}$ et le coefficient de régression linéaire correspondant à la même période.

\section{COURBES DANOISES :}

ESKEDAL (2) signale que des courbes de lactation standards sont distribuées, pour des buts pratiques de nutrition et de sélection, aux éleveurs de bétail danois.

\section{d) Traite du matin et du soir}

BARTLETT (9) donne les courbes de lactation moyennes pour les traites du matin et du soir de 97 vaches Shorthorn; 1 'intervalle de temps entre la traite du matin et celle du soir était de $8 \mathrm{~h} 3 / 4$ et de $5_{5} \mathrm{~h} 3 / 4$ entre celle du soir et celle du matin. Le rapport de ce $2^{\mathrm{e}}$ temps au $\mathrm{I}^{\mathrm{er}}$ était donc de $\mathrm{r}, 74$. Les courbes indiquent clairement la variation du rapport calculé 
des productions de la traite du matin à la traite du soir, prise comme unité. Ce rapport était de $I, 47$ dans la phase ascendante et au maximum de la lactation ; il croissait régulièrement jusqu'à $\mathrm{I}, 66$ au $8^{\mathrm{e}}$ mois de la lactation. EDWARDS (I4) a confirmé récemment cette différence dans l'allure

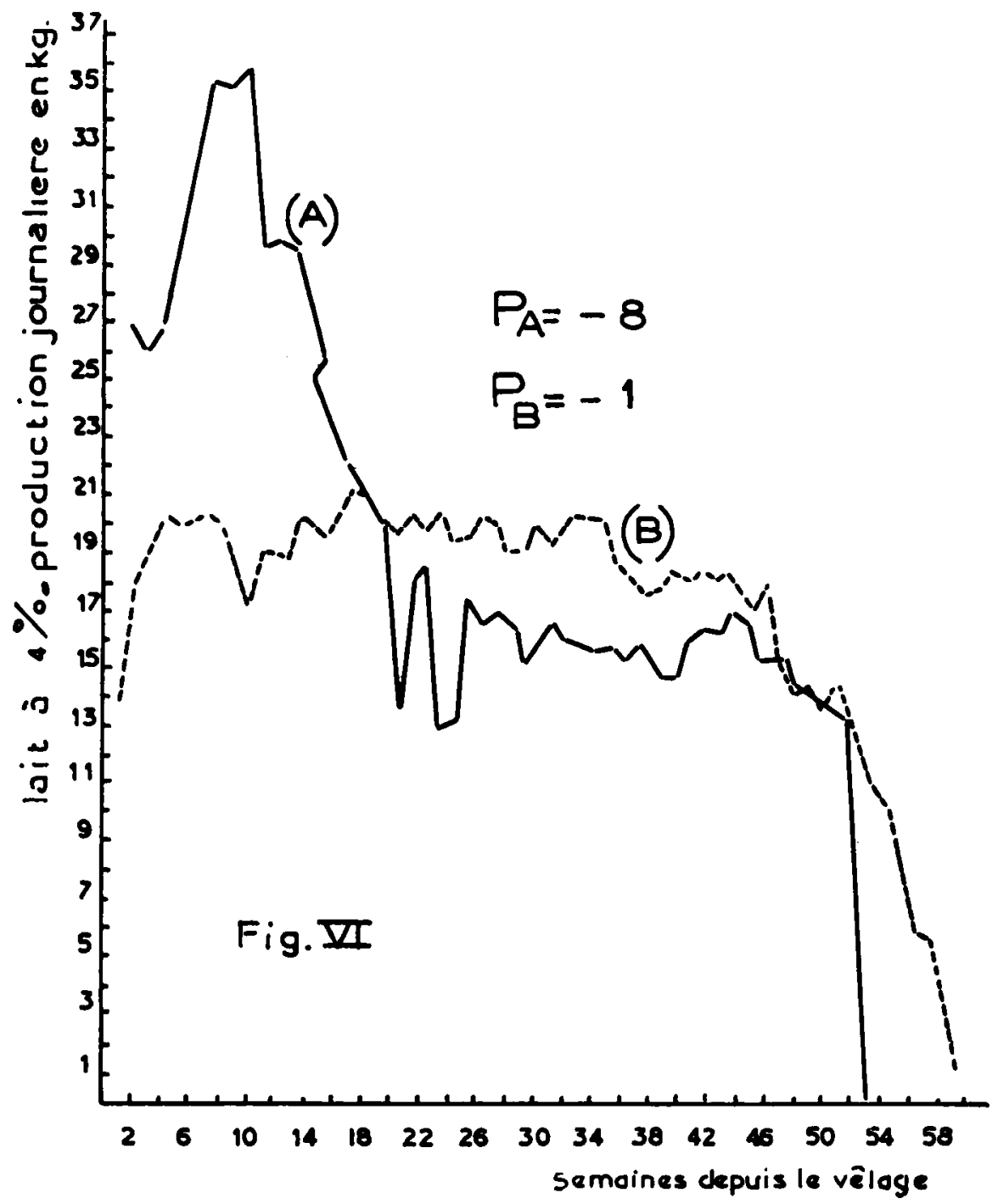

des graphiques de production correspondant aux traites du matin et du soir ; la courbe du soir est à partir $\mathrm{du} 45^{\mathrm{e}}$ jour pratiquement linéaire en coordonnées semi-logarithmiques, ce qui indique, selon lui, un déclin régulier dans 1'activité secrétrice; la courbe des productions du matin ne lui est parallèle qu'à partir du $180^{e}$ jour ; avant cette date la pente de la courbe représentative en coordonnées semi-logarithmiques est plus 
faible; cela est dûu, selon l'auteur, à l'effet néfaste de la pression intramammaire sur l'activité de la glande. Nous avons schématisé les résultats de l'auteur sur la figure 7 , l'aire hachurée correspondant à la perte de lait

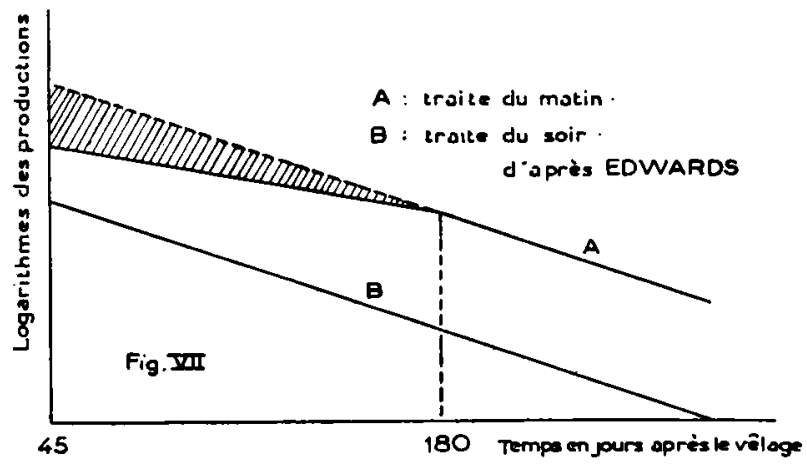

due à cette pression. D'ailleurs, les courbes de production correspondant à des animaux qui sont traits trois fois, sont exactement parallèles, selon EDWARDS, qui conclut qu'une courbe de production dans la soirée, résume mieux l'activité physiologique de la glande.

\section{C) Discussion des travaux antérieurs}

Beaucoup d'opinions ont été émises sur l'époque à laquelle le maximum de production est atteint au cours de la lactation; par suite de différences de nature génétique entre races, - Mc CANDLISH (6) -, entre animaux d'une même race, - Csukas (I5) - , et de diverses conditions de milieu et d'entretien des vaches, - fréquence des traites, par exemple ; TuRnER et coll. (5) - , la phase ascendante de la lactation présente en définitive une grande variabilité dans sa durée. Pour connaître de façon suffisamment précise cette période de production, des contrôles laitiers quotidiens, ou au moins hebdomadaires seraient nécessaires. Sinon durée et maximum de cette phase ne sont que très grossièrement estimés. Seuls peuvent être retenus, par conséquent, les résultats obtenus en station à 1'aide de troupeaux expérimentaux. Mais une étude sérieuse de la phase ascendante de la lactation, qui présenterait d'ailleurs un réel intérêt biologique, tant génétique que physiologique, reste à faire.

La phase de décroissance de la lactation a, par contre, été beaucoup mieux étudiée. Un grand nombre de formules, d'équations, d'indices, a été proposé pour la caractériser, les buts poursuivis par chacun des auteurs étant, la plupart du temps, très différents. C'est en fonction d'un objectif déterminé qu'il faut, par conséquent, rechercher le meilleur mode de représentation de la décroissance de la lactation.

Comme le fait remarquer PONTECORvo (27), pour des buts pratiques, la représentation graphique est non seulement suffisante, mais certaine- 
ment la plus intéressante, parce que la plus suggestive. Ainsi, le contrôle de 1'alimentation dans une étable par 1'examen des courbes de lactation est particulièrement fructueux. La courbe théorique à laquelle on peut se référer est une courbe exponentielle ; c'est le cas pour les fiches individuelles de contrôle laitier utilisées en France. I,es faisceaux de courbes qu'on y représente correspondent à des productions qui, partant de maxima variés, suivent une décroissance exponentielle avec une chute mensuelle constante de ro \%. Or, il a été prouvé par différents auteurs une corrélation négative entre le maximum de production journalière et la persistance de la lactation; c'est dire qu'on ne peut admettre le même pourcentage mensuel de décroissance pour les vaches à faible ou forte pointe de production. L'indice P de NIELSEN (45) est un moyen pratique pour adapter la phase ascendante de la lactation à une régression linéaire, sans le moindre calcul. Il serait peut-être intéressant de combiner les deux méthodes en caractérisant par un indice $\mathrm{P}$ les droites construites en coordonnées semi-logarithmiques, représentant les courbes réelles de production ; la représentation exponentielle de la phase de décroissance est, en effet, admise par la plupart des auteurs.

A des fins de sélection, pour caractériser la persistance, de nombreux auteurs ont calculé divers rapports de production correspondant à des périodes différentes de la lactation. A notre sens, les indices de persistance qui font intervenir la production maximum - S. F. de SANDERs $(35,36)$; indice de Turner (3I) ; coefficient $G$ de Fredericksen et OsterGaARD (37) - sont difficilement acceptables, car, dans la pratique courante du contrôle laitier, avec enregistrement mensuel des performances, ce maximum est connu de façon beaucoup trop imprécise. BARTIETT (46) ne signale-t-il pas que la variabilité des productions journalières est très importante au cours du I ${ }^{\text {er }}$ mois de lactation. De même, la méthode de CARLYLE et WOLL (38) ne peut être employée dans la pratique car on ne peut envisager des contrôles laitiers à semaine fixe dans l'exploitation normale des troupeaux. Le procédé employé par BRUUN (39) ne donne pas de valeur numérique pour la persistance. Les méthodes utilisées par Terho (40), Johansson et Hansson (42) si elles sont intéressantes et pratiques, manquent par trop de signification biologique; la phase ascendante, avec ses propres caractéristiques, se trouve incluse, soit dans la production durant les 5 premiers mois, soit dans la production des IOo premiers jours. Le calcul préconisé par PETERSEN et Ludwick (43) conduit à multiplier les divisions au cours de la phase de décroissance ; ces divisions, dont la durée est fixée arbitrairement à 80 jours, sont comptées à partir du $4^{8}$ jour de la lactation. On aboutit alors, selon nous, à des expressions dont la complexité pratique ne justifie plus de ne pas recourir à une expression mathématique. L'indice proposé récemment par MAHADEVAN (44) a un réel intérêt biologique, tout en étant d'un cal- 
cul facile; mais les réserves que nous avons faites sur l'utilisation de la production globale enregistrée durant la phase ascendante - ici 70 jours - restent valables; cette production dans le cas de contrôles mensuels, est connue avec une trop large approximation.

Peu d'auteurs ont cherché à adapter la phase de décroissance à une courbe de régression statistiquement définie. L'examen de courtes périodes explique les interprétations linéaires de BonNier (I8) et NIELSEN (45). Gaines (I7), puis Gooch (26) et Pontecorvo (27) admettant une décroissance exponentielle de la production, caractérisent la persistance soit par l'exposant $k$ de l'exponentielle, GaINES (I7) ; par le paramètre $e^{b}$, Gooch (26) ; par l'expression IOo. $e^{k}{ }^{k}$, PONTECoRvo (27). Ces différents paramètres sont d'une utilisation difficile à des fins de sélection ; n'est-il pas préférable, alors, de faire la moyenne des pourcentages mensuels de persistance, en partant du contrôle mensuel où la production maximum a été enregistrée ? SikKA (28), à des fins d'études génétiques a introduit une nouvelle représentation mathématique : l'exponentielle parabolique ; son utilisation dans la pratique de la sélection, paraît difficile. Il est, en outre, intéressant de remarquer que cet auteur étudie la courbe de lactation dès le premier mois après le vêlage ; or, très souvent, comme 1'indiquent quelques-unes de ses courbes, les vaches n'ont pas atteint leur production maximum ; l'étude de la phase descendante est alors entachée d'erreur au départ.

Une critique peut, en général, être formulée sur le mode d'utilisation des résultats mensuels de contrôle laitier qui ne tiennent pas compte du temps exact qui sépare la date du contrôle de celle du vêlage. Dans ces conditions, tous les contrôles portant un même rang d'ordre correspondent, par hypothèse, à une même époque de la courbe de lactation. Des erreurs systématiques sont introduites par cette méthode, les contrôles mensuels n'étant jamais effectués à des dates fixes.

Enfin, la majorité des travaux antérieurs a été poursuivie sur des courbes de lactation construites en lait à $4 \%$. Leur intérêt du point de vue énergétique ne saurait être méconnu, car la courbe de lactation, représente alors, selon GaINES, "le rendement réel de la mamelle ". Mais des études physiologiques et génétiques nécessitent la connaissance des productions réelles.

L'analyse détaillée des courbes de lactation semble nécessaire, d'autre part, avant toute étude physiologique intéressant la production laitière. Elle peut permettre de faire ressortir l'évolution des mécanismes physiologiques de sécrétion, de montrer l'influence des conditions de milieu, notamment d'hygiène et d'alimentation sur la production, et d'envisager leur contrôle dans la pratique de l'élevage.

Le but de cette étude est de définir la meilleure forme d'expression mathématique de la phase descendante de la lactation. 


\section{$2^{\circ}$ ETUDE EXPERIMENTALE}

\section{A) Matériel d'étude}

Le Syndicat de Contrôle Laitier et d'Élevage de Seine-et-Marne, dont nous suivons régulièrement l'enregistrement des lactations, l'état sanitaire et l'alimentation des animaux contrôlés, nous a permis d'étudier environ I 250 lactations obtenues en I949, dans 92 étables de vaches Française-Frisonne Pie Noire.

Nous n'avons pas retenu l'ensemble de ces données afin d'éliminer les influences perturbatrices consécutives à des épizooties et à des fautes d'alimentation. Un état sanitaire défectueux ou une alimentation insuffisante sont, en effet, susceptibles d'entraîner des chutes anormales et passagères de production qui ne peuvent être imputées à des mécanismes physiologiques normaux ainsi qu'en témoignent les courbes de la figure 8 ,
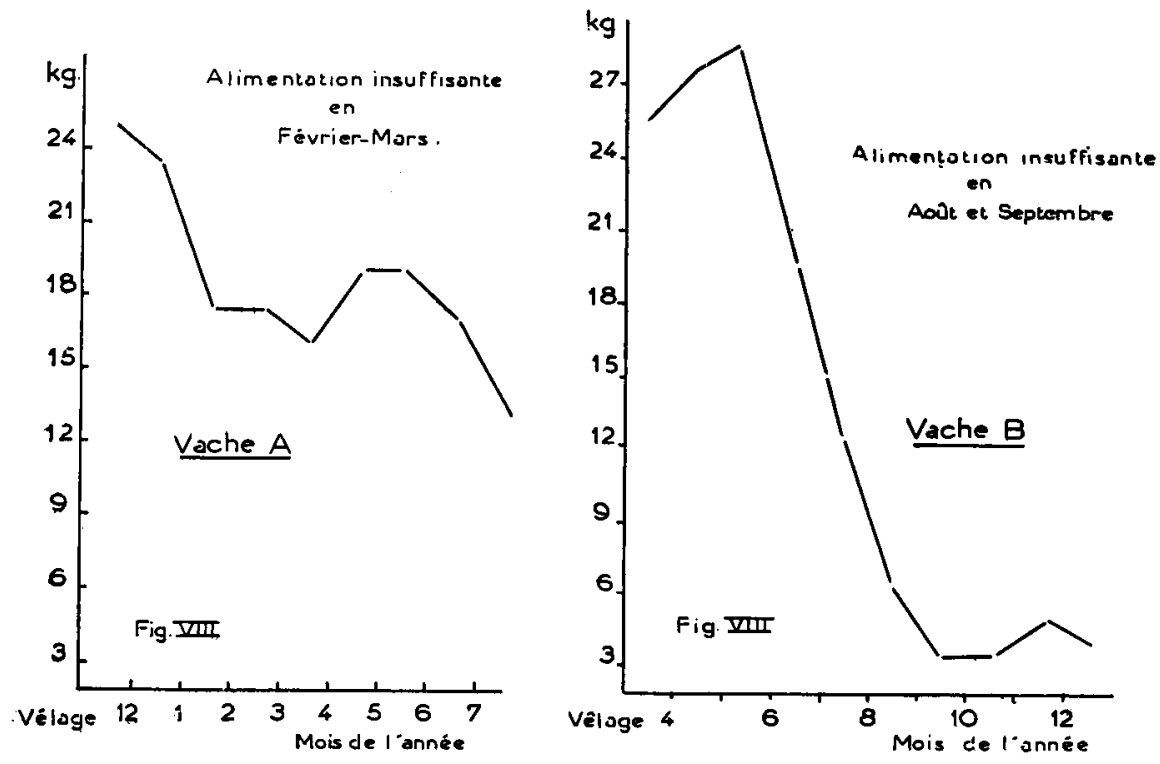

qui traduisent une sous-alimentation en fin d'hiver pour la vache $\mathrm{A}-$ due à une insuffisance en qualité et en quantité des ressources fourragères à la fin de la période de stabulation —, une sous-alimentation de la vache $B$, en juillet, août, septembre, imputable à la pauvreté des pâturages, pendant la sécheresse de l'été.

Nous n'avons pas gardé par ailleurs les lactations dont la durée était inférieure à 300 jours. Cette durée de 300 jours correspond à des vaches qui sont normalement entretenues dans les conditions pratiques de l'élevage, et qui vêlent chaque année après une période de repos mammaire 
de 2 mois. Une étude statistique préalable $\left({ }^{1}\right)$ nous a montré que sur 567 animaux, la durée moyenne de la lactation était, en I949, de 308 jours. Cette durée ne semble pas varier avec les années puisque nous avons obtenu, en I950, une durée moyenne de 305 jours, non significativement différente.

Ainsi avons-nous pu étudier, dans les I2 meilleures étables du Syndicat, I46 lactations obtenues en I949, correspondant à des vêlages répartis à peu près équitablement entre les 12 mois de l'année, et nous avons utilisé les données correspondantes pour construire une courbe moyenne de lactation. Les vaches étudiées effectuaientleur $2^{\mathrm{e}}, 3^{\mathrm{e}}$ ou $4^{\mathrm{e}}$ lactation, étaient traites 2 fois par jour, et saillies en moyenne r 40 jours après le vêlage.

\section{B) Méthode utilisée}

Les opérations de contrôle laitier étaient effectuées dans chaque étable à des intervalles de temps compris entre 26 et 33 jours. Lue premier contrôle ne pouvait avoir lieu avant le $5^{\mathrm{e}}$ jour suivant le vêlage.

Nous avons construit graphiquement, pour chaque vache, la courbe de lactation en portant en ordonnées la production en $\mathrm{kg}$ obtenue aux différents contrôles et en abscisses leur date en jours écoulés depuis le vêlage.

La courbe moyenne correspondant aux I 46 lactations a été obtenue à partir des courbes individuelles précédentes. L,es ordonnées de cette courbe sont des moyennes de production de io jours en Io jours.

Cette méthode nous a permis :

a) d'analyser, avec un maximum de précision, les lactations individuelles et de faire ressortir ainsi la grande variabilité graphique des courbes de lactation;

b) d'éliminer les erreurs qui auraient résulté du calcul de la moyenne des productions lors de contrôles de même rang d'ordre, sans tenir compte de leur date exacte à partir du vêlage. Avec la réglementation du contrôle laitier, le $3^{\mathbf{e}}$ contrôle pouvait par exemple, se situer $\mathrm{du} 59^{\mathrm{e}}$ au Io $8^{\mathrm{e}}$ jour suivant le vêlage.

Le présent mémoire a pour objet l'étude de la courbe moyenne.

\section{C) Étude de la courbe moyenne}

\section{a) Phase ascendante de la courbe de lactation}

Nos données ne nous permettaient pas d'entreprendre une étude suffisamment précise de la phase ascendante de lactation; nous avons

(i) En collaboration avec Mr Rossier, Ingểnieur Agronome, du Polytechnicum de Zurich. 
seulement étudié la phase descendante de la courbe, à partir du $60^{\mathbf{e}}$ jour après le vêlage, époque à laquelle $96 \%$ des animaux examinés avaient dépassé leur maximum de production.

\section{b) Phase descendante de la courbe de lactation}

I'examen de la courbe moyenne de décroissance de la production nous a conduits à distinguer 2 parties dans la phase descendante (fig. 9) :

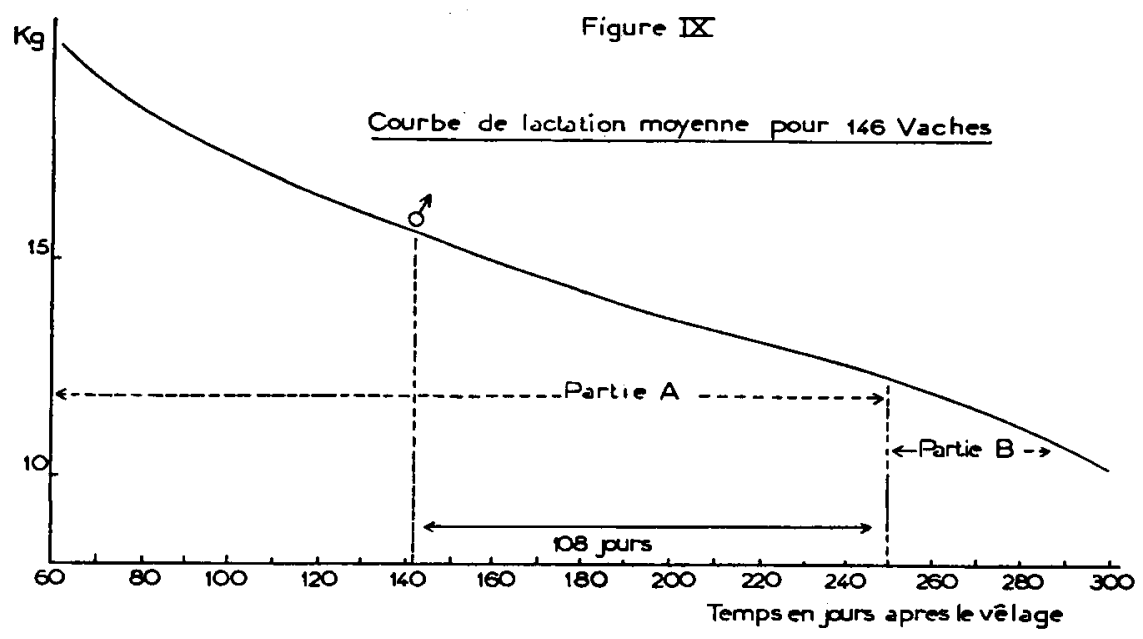

l'une (A), s'étendant du $60^{\mathrm{e}}$ au $25^{\circ}$ jour ; l'autre (B), du $250^{\mathrm{e}}$ au $300^{\mathrm{e}}$ jour, et à rechercher ultérieurement l'influence de la gestation sur la production laitière.

ÉTUDE STATISTIQUE DE LA COURBE DE LACTATION MOYENNE DU $60^{\circ}$ jour AU $25^{\circ}$ jOUR APRÈS LE VÊLAGE :

Ainsi, nous avons été amenés à rechercher la meilleture ligne de régression qui caractérise la courbe de lactation entre le $60^{\mathrm{e}}$ et le $25^{\mathrm{e}}$ jour après le vêlage (fig. Io),

Nous nous sommes limités à 4 hypothèses que nous avons testées avec nos propres données : la droite, l'exponentielle, l'hyperbole, la parabole.

Le tableau I résume l'ensemble de nos résultats :

\section{Hypothèse d'une régression linéaire :}

Si nous désignons par $y$ la production journalière, et par $x$ le temps jours depuis le vêlage. l'équation de la droite de régression de $y$ en $x$ est :

$$
\mathrm{Y}=\mathrm{A}+\mathrm{B}(x-\bar{x})
$$


TABLEAU I

\begin{tabular}{|c|c|c|c|c|c|}
\hline $\begin{array}{c}\text { Classes. } \\
\text { Temps en jours } \\
\text { écoulés depuis } \\
\text { le vêlage }\end{array}$ & $\begin{array}{c}\text { Productions } \\
\text { moyennes } \\
\text { observées pour } \\
\text { r46 vaches } \\
\text { en } \mathrm{kg}\end{array}$ & $\begin{array}{l}\text { Ajustement } \\
\text { linéaire. } \\
\text { Productions } \\
\text { en } \mathrm{kg}\end{array}$ & $\begin{array}{c}\text { Ajustement } \\
\text { exponentiel. } \\
\text { Productions } \\
\text { en kg }\end{array}$ & $\begin{array}{l}\text { Ajustement } \\
\text { parabolique. } \\
\text { Productions } \\
\text { en kg }\end{array}$ & $\begin{array}{l}\text { Ajustement } \\
\text { hyperbolique. } \\
\text { Productions } \\
\text { en kg }\end{array}$ \\
\hline $60 \ldots \ldots \ldots \ldots$ & I9,806 & 19,228 & 19,500 & $19,78 \mathrm{I}$ & $2 \mathrm{I}, \mathrm{I} 75$ \\
\hline $70 \ldots \ldots \ldots \ldots$ & 19,204 & 18,827 & 19,002 & 19,206 & 19,655 \\
\hline $80 \ldots \ldots \ldots \ldots$ & 18,635 & 18,427 & $18,5 \mathrm{I} 7$ & 18,650 & $18,5 \mathrm{I} 4$ \\
\hline $9 \circ \ldots \ldots \ldots \ldots$ & 18,042 & $\mathbf{1} 8,027$ & I 8,044 & I 8, I 4 & 17,626 \\
\hline 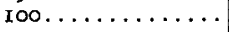 & 17,540 & 17,626 & 17,583 & I 7,597 & 16,918 \\
\hline IIo $\ldots \ldots \ldots \ldots$ & 17,138 & 17,226 & $17, I_{34}$ & 17,100 & 16,337 \\
\hline $120 \ldots \ldots \ldots \ldots$ & $16,68 \mathrm{r}$ & 16,826 & 16,696 & 16,622 & 15,853 \\
\hline I $30 \ldots \ldots \ldots \ldots \ldots$ & 16,253 & 16,425 & 16,270 & 16,163 & I 5,444 \\
\hline $140 \ldots \ldots \ldots \ldots$ & r 5,773 & $x 6,025$ & 15,856 & 15,724 & I 5,093 \\
\hline $1_{5}{ }^{\circ} \ldots \ldots \ldots \ldots$ & $15,3 \circ 5$ & 15,624 & $I_{5}, 45^{\circ}$ & 15,304 & $\mathrm{I} 4,789$ \\
\hline $160 \ldots \ldots \ldots \ldots$ & $14,85^{6}$ & 15,224 & I 5,055 & I 4,904 & $14,5^{2} 3$ \\
\hline I7o $\ldots \ldots \ldots \ldots$ & $\mathrm{I} 4,47 \mathrm{I}$ & I 4,824 & $14,67 \mathrm{I}$ & 14,523 & 14,288 \\
\hline $180 \ldots \ldots \ldots \ldots$ & I4,137 & 14,423 & 14,396 & 14,161 & I4,079 \\
\hline I $90 \ldots \ldots \ldots \ldots$ & 13,792 & 14,023 & $\mathrm{r}_{3,934}$ & 13,820 & 13,892 \\
\hline $200 \ldots \ldots \ldots \ldots \ldots$ & I 3,494 & 13,623 & I 3,576 & I 3,497 & $1_{3,724}$ \\
\hline $210 \ldots \ldots \ldots \ldots$ & I 3,184 & 13,222 & I 3,229 & I3,I93 & 13,572 \\
\hline $220 \ldots \ldots \ldots \ldots \ldots$ & I 2,9 I 2 & 12,822 & ז 2,894 & $+2,909$ & I 3,434 \\
\hline $230 \ldots \ldots \ldots \ldots$ & 12,684 & 12,422 & $12,55^{2}$ & 12,645 & I 3,308 \\
\hline $240 \ldots \ldots \ldots \ldots$ & I 2,428 & $12,02 \mathrm{I}$ & $\mathrm{I} 2,24 \mathrm{I}$ & 12,400 & I 3,292 \\
\hline $250 \ldots$ & I $2,15 \mathrm{I}$ & $I I, 621$ & I I,929 & I 2,174 & I 3,086 \\
\hline
\end{tabular}

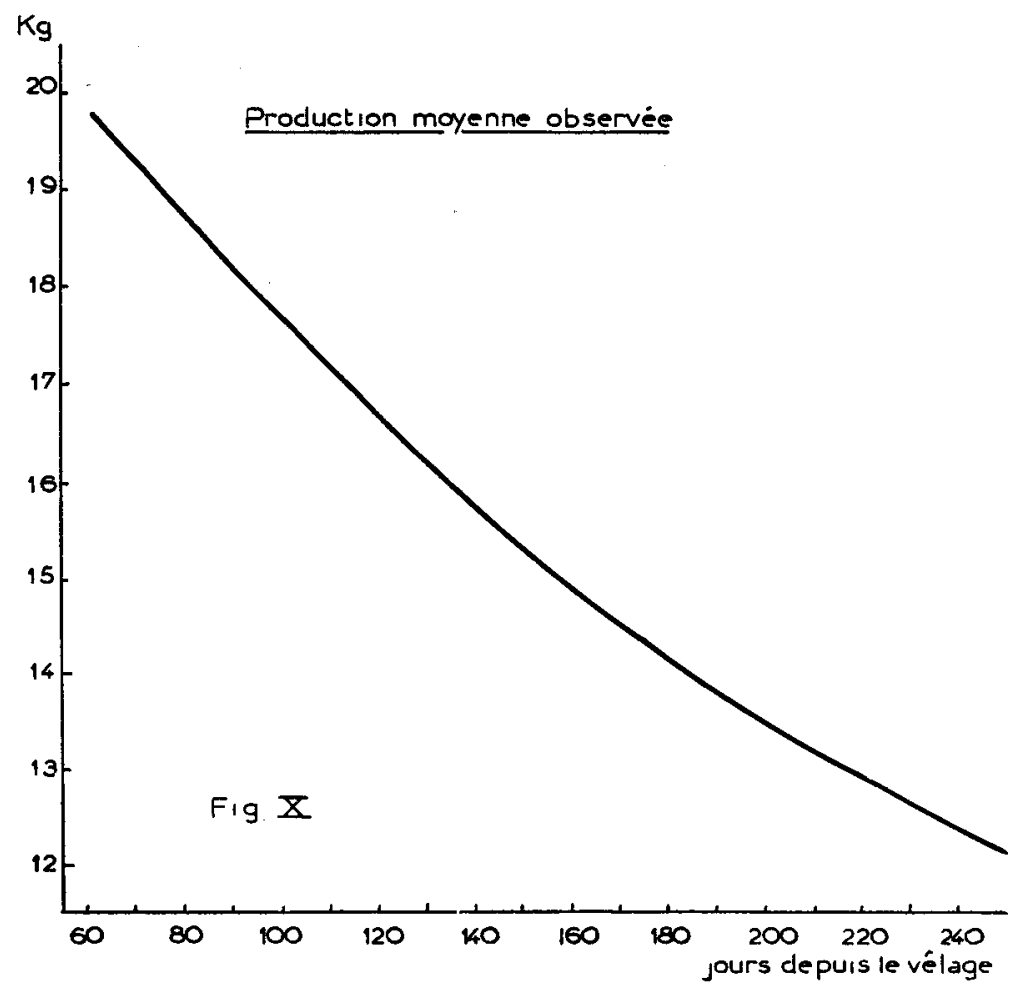


dans laquelle Y représente les valeurs d'ajustement,

$$
\mathrm{A}=\bar{y}=25,424 \text { et } \quad \bar{x}=\frac{60+70+\cdots+240+250}{20}=\mathrm{I} 55
$$

La valeur du coefficient de régression $\mathrm{B}$ est donnée par la relation :

$$
\mathrm{B}=\frac{\sum\left(x_{i}-\bar{x}\right)\left(y_{i}-\bar{y}\right)}{\sum\left(x_{i}-\bar{x}\right)^{2}}
$$

L'équation de la droite de régression correspondant aux valeurs moyennes des différentes classes, est alors :

$$
\begin{aligned}
& \mathrm{Y}=\mathrm{I} 5,424-0,04004(x-\mathrm{I} 55) \\
& \mathrm{Y}=2 \mathrm{I}, 63-0,04004 x
\end{aligned}
$$

Cette droite est représentée sur la figure II.

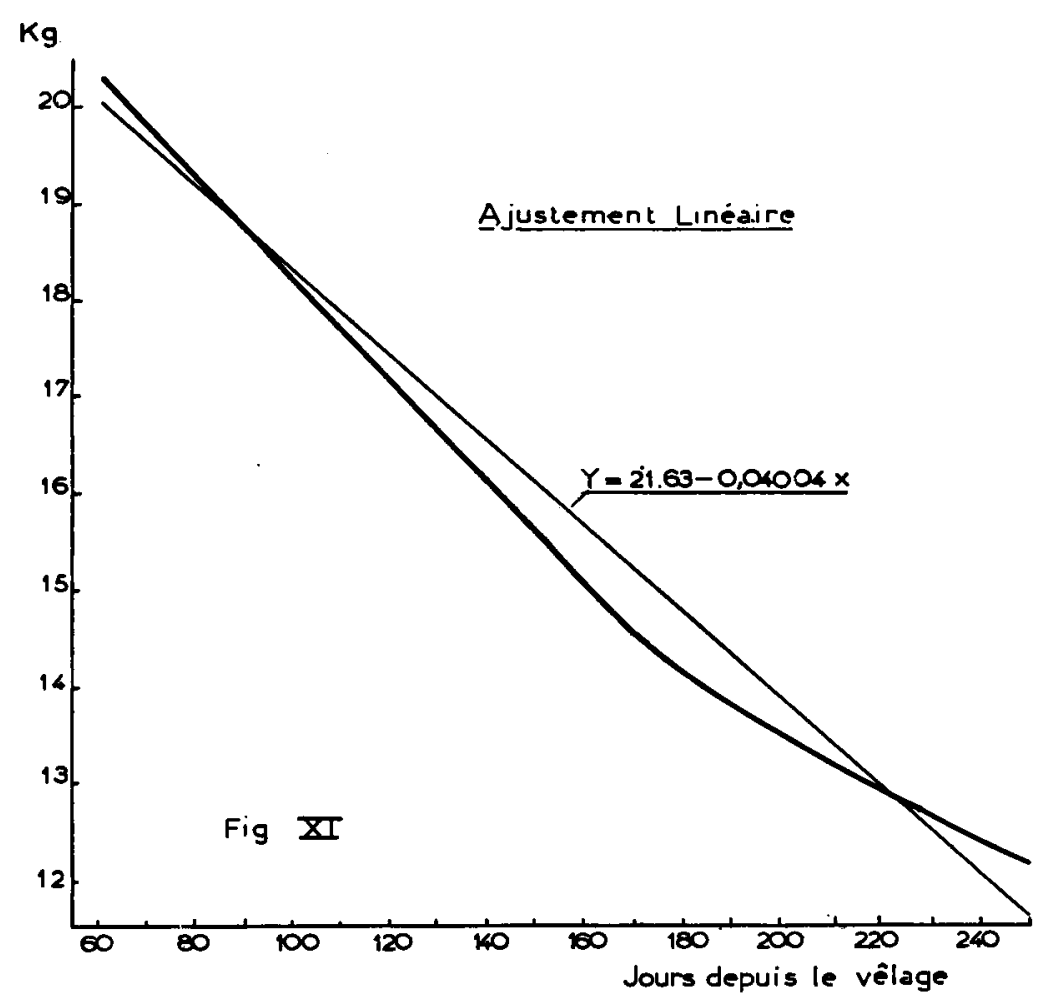

Erreurs d'échantillonnage :

Pour tenir compte du nombre limité des animaux étudiés, nous sommes conduits à calculer les erreurs d'échantillonnage commises sur les paramètres de la droite de régression. 
L'erreur commise sur le paramètre A est donnée par la formule :

$$
s_{A}=\sqrt{\frac{\sigma^{2}}{\bar{N}}}
$$

où $\mathrm{N}$ désigne le nombre total d'observations et $\sigma^{2}$ la variance des déviations des valeurs observées par rapport à la ligne de régression.

Remarquons que, malgré l'égalité de $\mathrm{A}$ et $\bar{y}$, l'erreur-type de $\mathrm{A}$ n'est pas celle de $\bar{y}$ pour laquelle il faudrait calculer la variance générale des productions ; A étant un paramètre de position et non de pente, il est donc logique de négliger les variations de $y$ imputables aux variations de $x$.

La meilleure estimation de cette variance $\sigma^{2}$ est :

$$
\mathrm{S}^{2}=\frac{\sum(y-\mathrm{Y})^{2}}{\mathrm{~N}-2},
$$

où $y$ désigne les productions réelles observées, Y les valeurs d'ajustement correspondantes ; $\mathrm{N}-2$ est le nombre de degrés de liberté, puisque deux relations ont été utilisées entre les $\mathrm{N}$ mesures, pour le calcul de $\mathrm{A}$ et $\mathrm{B}$.

Pour calculer $\Sigma(y-\mathrm{Y})^{2}$, nous utilisons la relation

$$
\sum\left(y_{i}-Y_{i}\right)^{2}=\sum\left(y_{i}-\overline{y_{i}}\right)^{2}+I_{4} 6 \sum_{I}^{20}\left(\overline{y_{i}}-Y_{i}\right)_{i}^{2}
$$

nous avons évalué $\Sigma\left(y_{i}-\bar{y}_{i}\right)^{2}$ à partir des données de la classe 60 , où la variabilité des productions est la plus élevée.

D'autre part, nous calculons $\sum_{I}^{20}\left(\bar{y}_{i}-Y_{i}\right)^{2}=I, 685$,

$$
\sum_{\mathrm{I}}^{20}\left(\overline{y_{i}}-\mathrm{Y}_{i}\right)^{2}=\mathrm{I}, 685
$$

d'où

$$
\sum(y-\mathrm{Y})^{2}=\mathrm{II} 2.004
$$

et en définitive :

$$
s_{\Delta}= \pm 0, \mathrm{x} 2 \text {. }
$$

L'erreur d'échantillonnage commise sur le paramètre $B$ est évaluée par la formule :

$$
s_{\mathrm{B}}=\sqrt{\frac{\sigma^{2}}{\sum(x-\bar{x})^{2}}}
$$

$\sigma^{2}$ représente toujours la variance des déviations des valeurs observées par rapport à la ligne droite théorique, dont nous avons calculé déjà l'estimation $\mathrm{S}^{2}$. जif $\pm 0,002$.

Nous pouvons alors écrire l'équation de la droite de régression :

$$
\mathrm{Y}=2 \mathrm{I}, 63( \pm 0, \mathrm{I} 2)-0,04( \pm 0,002) x \text {. }
$$


Si nous exprimons le temps $t$ en mois écoulés depuis le vêlage, cette équation devient :

$$
\mathrm{Y}=2 \mathrm{I}, 63( \pm 0, \mathrm{x} 2)-\mathrm{I}, 2( \pm 0,06) t \text {. }
$$

Cette régression linéaire permet de penser que la production laitière baisse chaque mois entre le $60^{\mathrm{e}}$ et le $25^{\mathrm{e}}$ jour, d'une quantité constante moyenne égale à $\mathrm{I}, 2 \mathrm{~kg}$ chez les animaux étudiés.

Le coefficient $B$ est donc une mesure absolue de la décroissance mensuelle.

L'ordonnée à l'origine de cette droite de régression n'a, évidemment, aucune signification pratique, puisqu'elle représente la production fictive de la vache le jour du vêlage, origine des temps.

Il est possible de transformer cette équation en situant l'origine des temps 60 jours après le vêlage; nous avons alors, en désignant par $\mathrm{X}$ le temps en jours à partir de cette date:

et, en définitive :

$$
\mathrm{Y}=2 \mathrm{x}, 63-0,04(\mathrm{X}+60)
$$

$$
\mathrm{Y}=\mathrm{r} 9,23-0,04 \mathrm{X} .
$$

I 9,23 , représente la production théorique au $60^{\mathrm{e}}$ jour après le vêlage.

En désignant par $\mathrm{T}$ le temps en mois à partir de cette nouvelle origine, nous avons l'équation (6):

$$
\mathrm{Y}=\mathrm{I} 9,23( \pm 0, \mathrm{I} 2)-\mathrm{I}, 2( \pm 0,06 \mathrm{~T} \text {. }
$$

\section{Signification statistique de la régression linéaire}

Rappelons les bases théoriques du test de linéarité. Considérons la figure I2 où la droite de régression de $y$ en $x$ a été construite.

Soit $x_{i}$ une valeur de la variable indépendante ; $Y_{i}$, la valeur correspondante de $y$ pour l'ajustement linéaire; $\bar{y}_{i}$ la moyenne des valeurs de $y$ réellement observées dans la classe $x_{i}$; nous avons la relation suivante en valeurs algébriques :

$$
\overline{y_{i}}-\bar{v}=\left(\mathrm{Y}_{i}-\bar{y}\right)+\left(\overline{y_{i}}-\mathrm{Y}_{i}\right)
$$

Les termes $\mathrm{Y}_{i}-\bar{y}$ sont connus avec un seul degré de liberté car il suffit de connaître une valeur du type $Y_{i}$ pour que la droite de régression soit entièrement déterminée, et, par suite, toutes les autres différences $\mathrm{Y}_{i}-\bar{y}$.

En définitive, si nous a rons $k$ valeurs de classe de la variable indépendante, nous avons la relation suivante entre les degrés de liberté relatifs à chaque terme de l'égalité (I).

$$
k-\mathrm{I}=\mathrm{I}+(k-2) \text {. }
$$

Si la liaison de $y$ en $x$ était fonctionnelle, les valeurs $\left(\bar{y}_{i}-\mathrm{Y}_{i}\right)$ seraient évidemment nulles ;

Le test de linéarité revient à prouver que les différences de ce type peuvent être imputées à l'effet de seules causes fortuites alors que les différences $\left(\mathrm{Y}_{i}-\bar{y}\right)$ représentent l'effet d'une cause systématique de variation. 
En définitive, nous pouvons donc écrire :

d'où nous tirons :

$$
\boldsymbol{\Sigma}\left(\overline{y_{i}}-\bar{y}\right)^{2}=\boldsymbol{\Sigma}\left(\mathrm{Y}_{i}-\bar{y}\right)^{2}+\boldsymbol{\Sigma}\left(\overline{y_{i}}-\mathrm{Y}_{i}\right)^{2}
$$

$$
\boldsymbol{\Sigma}\left(\overline{y_{i}}-\bar{y}\right)^{2}=\mathrm{B}^{2} \boldsymbol{\Sigma}\left(x_{i}-\bar{x}\right)^{2}+\boldsymbol{\Sigma}\left(\overline{y_{i}}-\mathrm{Y}\right)^{2}
$$

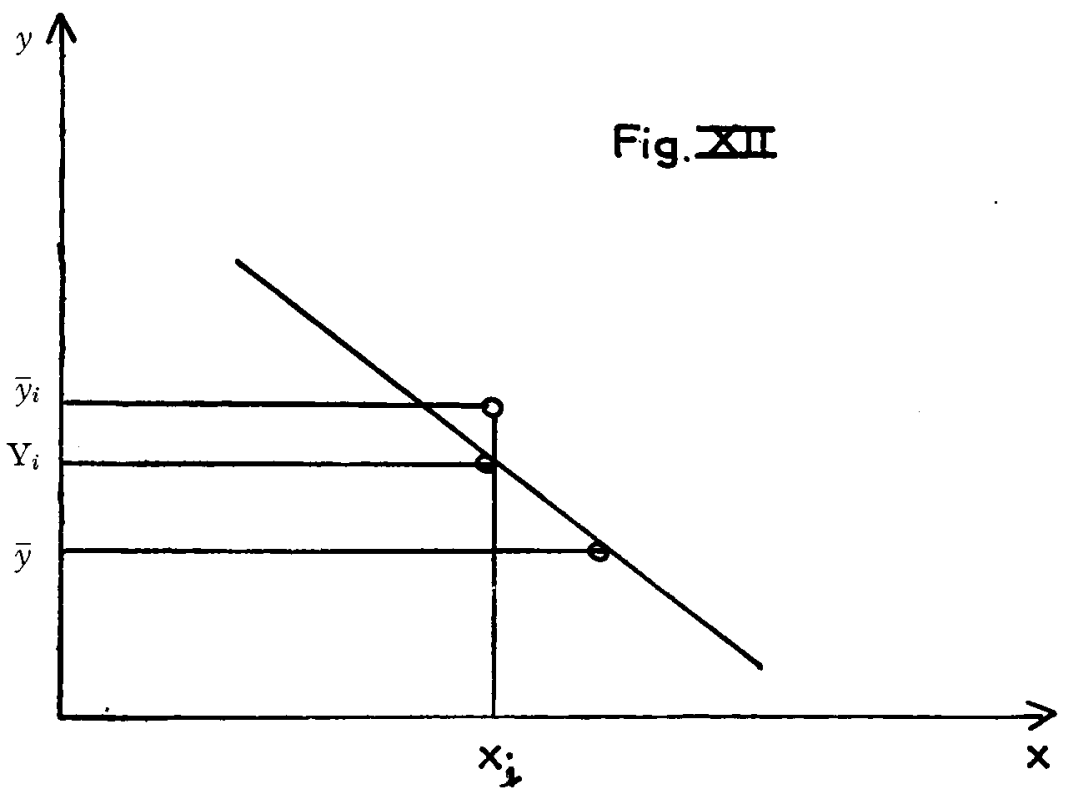

et pour les degrés de liberté :

$$
k-\mathrm{I}=\mathrm{I}+(k-2) .
$$

\begin{tabular}{|c|c|c|c|}
\hline Sources de la variation & Somme des carrés & Degrés de liberté & Variance \\
\hline Régression linéaire $\ldots \ldots \ldots \ldots \ldots$ & $\Sigma\left(x_{i}-\bar{x}\right)^{2}$ & I & - \\
\hline Déviations par rapport à la régression. . & $S\left(\bar{y}_{i}-Y_{i}\right)^{2}$ & $k-2$ & $s_{\mathrm{D}}^{2}=\frac{\mathrm{Y}\left(\bar{y}_{i}-\mathrm{Y}_{i}\right)^{2}}{k-2}$ \\
\hline Variation " entre classes" .......... & $\mathrm{Y}\left(\bar{y}_{i}-\bar{y}\right)^{\mathbf{2}}$ & $k-\mathbf{I}$ & $s_{\mathrm{E}}^{2}=\frac{\mathrm{Y}\left(\bar{y}_{i}-\bar{y}\right)^{2}}{k-\mathrm{I}}$ \\
\hline Variation "intraclasses $" \ldots \ldots \ldots \ldots$ & $\stackrel{\Sigma}{ }\left(y_{i}-\bar{y}_{i}\right)^{2}$ & $\Lambda^{\top}-k$ & $s_{\mathrm{I}}^{2}=\frac{Y\left(y_{i}-\bar{y}_{i}\right)^{2}}{N-k}$ \\
\hline Variation totale $\ldots \ldots \ldots \ldots \ldots \ldots$ & $\Sigma(y-y)^{2}$ & $N-I$ & $s_{\mathbf{T}}^{2}=\frac{\grave{y}(y-\bar{y})^{2}}{N-I}$ \\
\hline
\end{tabular}

Un résumé est donné par le tableau ci-dessous d'analyse de variance :

Application à l'étude de la courbe de lactation interprétée linéairement entre le $60^{\mathrm{e}}$ et le $250^{\mathrm{e}}$ jour

$$
s_{1}^{2}=13,8 \text {. }
$$

Testons cette variance $s_{\mathrm{r}}^{2}$ par rapport à la variance résiduelle qui représente l'action de causes fortuites. 
La variance $s_{D}^{2}$ doit être inférieure, égale, ou non significativement plus grande que la variance résiduelle $s_{\mathrm{I}}^{2}$.

Or

Done

$$
\begin{aligned}
& s_{\mathrm{I}}^{2}=24,6 \\
& s_{\mathrm{l},}^{2}<s_{\mathrm{I}}^{2} .
\end{aligned}
$$

L'ajustement linéaire serait donc statistiquement valable. Il nous faut noter cependant qu'en adaptant nos données expérimentales à une droite, nous commettons des erreurs systématiques, car la courbe expérimentale dans sa partie moyenne est toujours en dessous de la droite, 1'inverse se produisant pour les parties extrêmes (fig. I I).

Nous avons donc cherché à tester d'autres hypothèses de régression.

\section{Hypothèse d'une régression exponentielle}

Pour établir la ligne de régression exponentielle qui traduit dans les meilleures conditions la courbe moyenne de lactation étudiée, nous sommes conduits à représenter cette dernière en coordonnées semi-logarith miques et à étudier la variation des logarithmes décimaux de la production, en fonction du temps.

Dans ce nouveau système de coordonnées 1'équation de la droite de régression est de la forme :

et avec nos données

$$
\log \mathrm{Y}=\mathrm{A}+\mathrm{B}(x-\bar{x})
$$

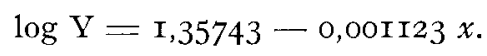

L'équation de l'exponentielle correspondante est alors :

$$
\mathrm{Y}=22,774 \cdot \mathrm{IO}^{-\mathbf{0 , 0 0 1 1 2 3} x}
$$

dans laquelle $Y$ et $x$ représentent respectivement, la production journalière en $\mathrm{kg}$ et le temps en jours.

Les erreurs d'échantillonnage sur chacun des coefficients de la droite de régression, en coordonnées semi-logarithmiques, se calculent comme précédemment et s'évaluent à :

$$
\begin{aligned}
& s_{\mathrm{A}}= \pm 0,0026 \\
& s_{\mathrm{B}}= \pm 0,00005 .
\end{aligned}
$$

L'équation de la droite de régression s'écrit alors :

$$
\log \mathrm{Y}=\mathrm{I}, 35743( \pm 0,0026)-0,00 \mathrm{II} 2( \pm 0,00005) x
$$

et l'équation de l'exponentielle :

$$
\mathrm{Y}=22,77( \pm 0, \mathrm{I} 3) \cdot \mathrm{IO}^{-0,00112( \pm 0,00005) x}
$$

En prenant pour origine des temps $1 \mathrm{e} 6 \mathrm{o}^{\mathrm{e}}$ jour après le vêlage, nous obtenons l'équation suivante :

$$
\mathrm{Y}=\mathrm{I} 9,5( \pm 0, \mathrm{I} 3) \cdot \mathrm{IO}^{-0,00112( \pm 0,00005) \mathrm{X}}
$$

I9,5 représente la production théorique au $60^{\mathrm{e}}$ jour après le vêlage. 
$\mathrm{Si}$ nous comptons le temps en mois à partir de cette origine, 1'exponentielle devient :

$$
\mathrm{Y}=\mathrm{I} 9,5( \pm 0, \mathrm{I} 3) \cdot 10^{-0,0337( \pm 0,0015) \mathrm{T}}
$$

L'hypothèse d'une régression exponentielle nous conduit à supposer que le pourcentage de la production d'un mois à celle du mois précédent est constante, et égale à :

$$
\mathrm{IO}^{-0,0327( \pm 0,0015)}=0,925 \pm 0,003 \text {. }
$$

Valeur statistique de la régression exponentielle

Nous pouvons, comme précédemment, calculer la variance des déviations des valeurs expérimentales par rapport à la régression exponentielle théorique ; or, avec 1'ajustement exponentiel $\Sigma\left(\bar{y}_{i}-\mathrm{Y}_{i}\right)^{2}=0,444$.

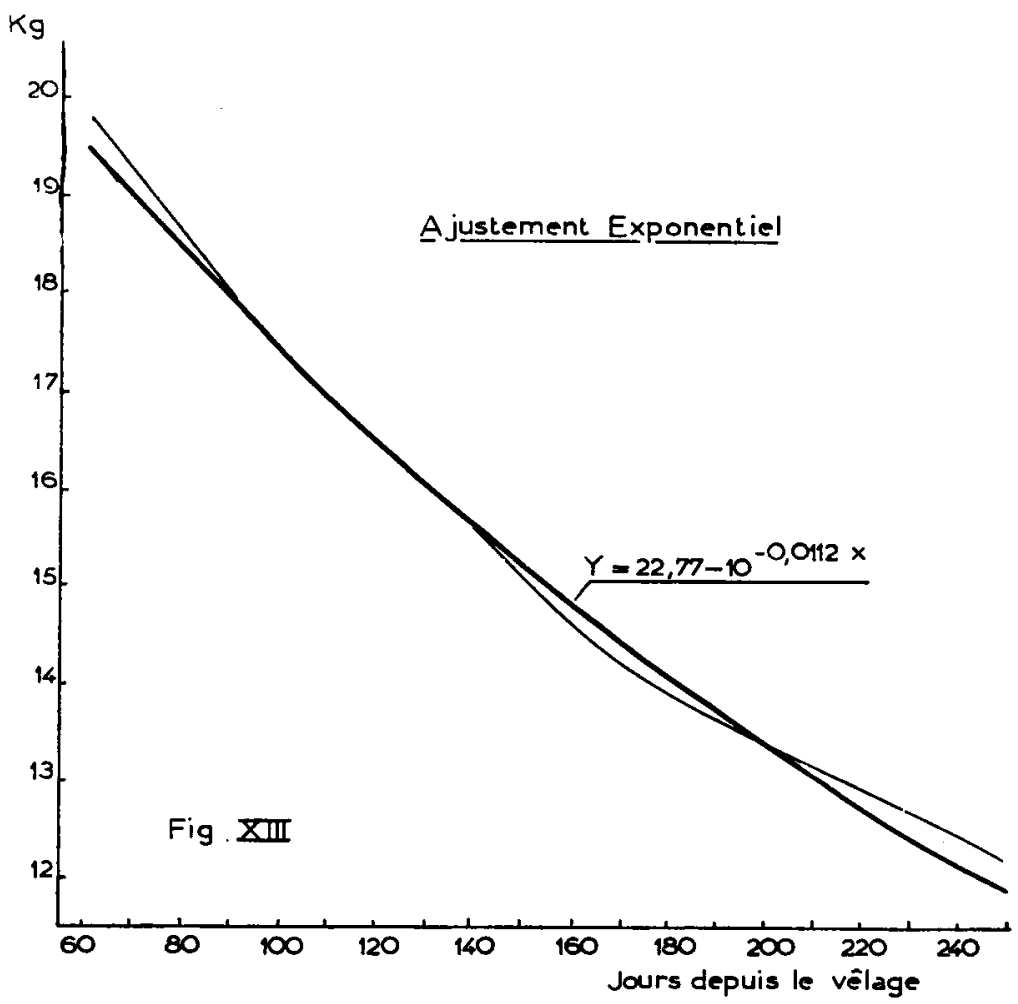

$s_{\mathbf{D}}^{2}$ sera donc inférieure à la valeur que nous avons calculée en admettant l'hypothèse d'une décroissance linéaire. L'hypothèse d'une régression exponentielle de la production laitière est vérifiée $a$ fortiori, car :

$$
\begin{array}{ll}
s_{1}^{2} \text { (régression exponentielle) } & <s_{\mathrm{D}}^{2} \text { (régression linéaire) } \\
\text { et } \quad s_{1}^{2} \text { (régression linéaire) } & <s_{\mathrm{I}}^{2} \text { (variance " intraclasses ») }
\end{array}
$$

Toutefois, il est intéressant de remarquer, ainsi qu'en témoigne la figure $\mathrm{r}_{3}$, qu'en admettant l'hypothèse d'une décroissance exponentielle 
de la production laitière, des erreurs systématiques sont commises. La courbe expérimentale, dans sa partie moyenne, est toujours en dessous de l'ajustement exponentiel ; l'inverse se produit dans les parties extrêmes de la courbe. Ce fait nous conduit à formuler d'autres hypothèses.

\section{Hypothèse d'une régression hyperbolique}

Nous devons chercher à ajuster nos données expérimentales à une régression hyperbolique de la forme :

$$
\mathrm{Y}=\frac{\mathrm{A}}{\mathrm{x}}+\mathrm{B}
$$

$\mathrm{A}$ et $\mathrm{B}$ sont des coefficients à déterminer d'après nos données.

La meilleure représentation hyperbolique de nos données sera celle qui rendra $\boldsymbol{\Sigma}\left(\bar{y}_{i}-\mathrm{Y}_{i}\right)^{2}$ minimum ;

$\bar{y}_{i}$ sont les productions moyennes observées; $\mathrm{Y}_{i}$ sont les valeurs théoriques d'ajustement.

Rendre $\Sigma\left(\bar{y}_{i}-\mathrm{Y}\right)^{2}$ minimum revient à rendre minimum :

$$
\left.\sum\left[\overline{y_{i}}-\left(\frac{\mathrm{A}}{\bar{x}_{i}}+\mathrm{B}\right)\right]^{2} \quad \text { ou } \quad \sum\left[\left(\overline{y_{i}}-\mathrm{B}\right)-\overline{\mathrm{A}}\right]_{\bar{i}}\right]^{2}
$$

et en désignant $\left(\bar{y}_{i}-\mathrm{B}\right)$ par $z_{i}$

C'est-à-dire :

$$
\sum\left(\bar{z}_{i}-\frac{\mathrm{A}}{x_{i}}\right)^{2} \text { minimum. }
$$

$$
\mathrm{U}=\sum z_{i}^{2}-2 \mathrm{~A} \sum \overline{\bar{z}_{i}}+\mathrm{A}^{2} \sum \frac{\mathrm{I}}{\bar{x}_{i}^{2}} \text { minimum. }
$$

A cette fin, nous annulons les dérivées de cette expression, par rapport à chacun des coefficients, soit :

$$
\frac{d \mathrm{U}}{d \mathrm{~A}}=0 ; \quad \frac{d \mathrm{U}}{d \mathrm{~B}}=0 .
$$

Nous obtenons alors ces deux équations linéaires en $\mathrm{A}$ et $\mathrm{B}$ :

$$
\begin{aligned}
& \mathrm{A}=\frac{\sum \frac{\overline{y_{i}}}{\bar{x}_{i}}-\mathrm{B} \sum \frac{\mathrm{I}}{\bar{x}_{i}}}{\sum \frac{\mathrm{I}}{x_{i}^{2}}} \\
& \mathrm{~B}=\bar{y}-\overline{\mathrm{A}} \sum \frac{\mathrm{I}}{\mathrm{N}} .
\end{aligned}
$$

Nous avons là un système de deux équations du premier degré à deux inconnues qu'il est facile de résoudre.

Nos données expérimentales nous fournissent pour ces 2 paramètres, les 2 valeurs suivantes:

$$
\left\{\begin{array}{l}
A=638,66 \\
B=10,53 .
\end{array}\right.
$$


Et pour le meilleur ajustement hyperbolique, l'équation

$$
\mathrm{Y}=\frac{638,6}{x}+\mathrm{ro,531}
$$

dans laquelle $\mathrm{Y}$ représente la production en $\mathrm{kg}$ et $x$ le temps en jours à partir du vêlage (fig. I4).

\section{Valeur statistique de l'ajustement hyperbolique}

Nous calculons $s_{\mathbf{D}}^{2}$, variance des déviations des productions observées aux productions théoriques d'ajustement.

$$
\sum\left(\overline{y_{i}}-\mathrm{Y}_{i}\right)^{2}=7,55 \quad \text { et } \quad s_{\mathrm{D}}^{2}=6 \mathbf{I}, 2
$$

Nous avons calculé précédemment la variance " intraclasses " estimée à partir de nos données et égale à 24,6 .

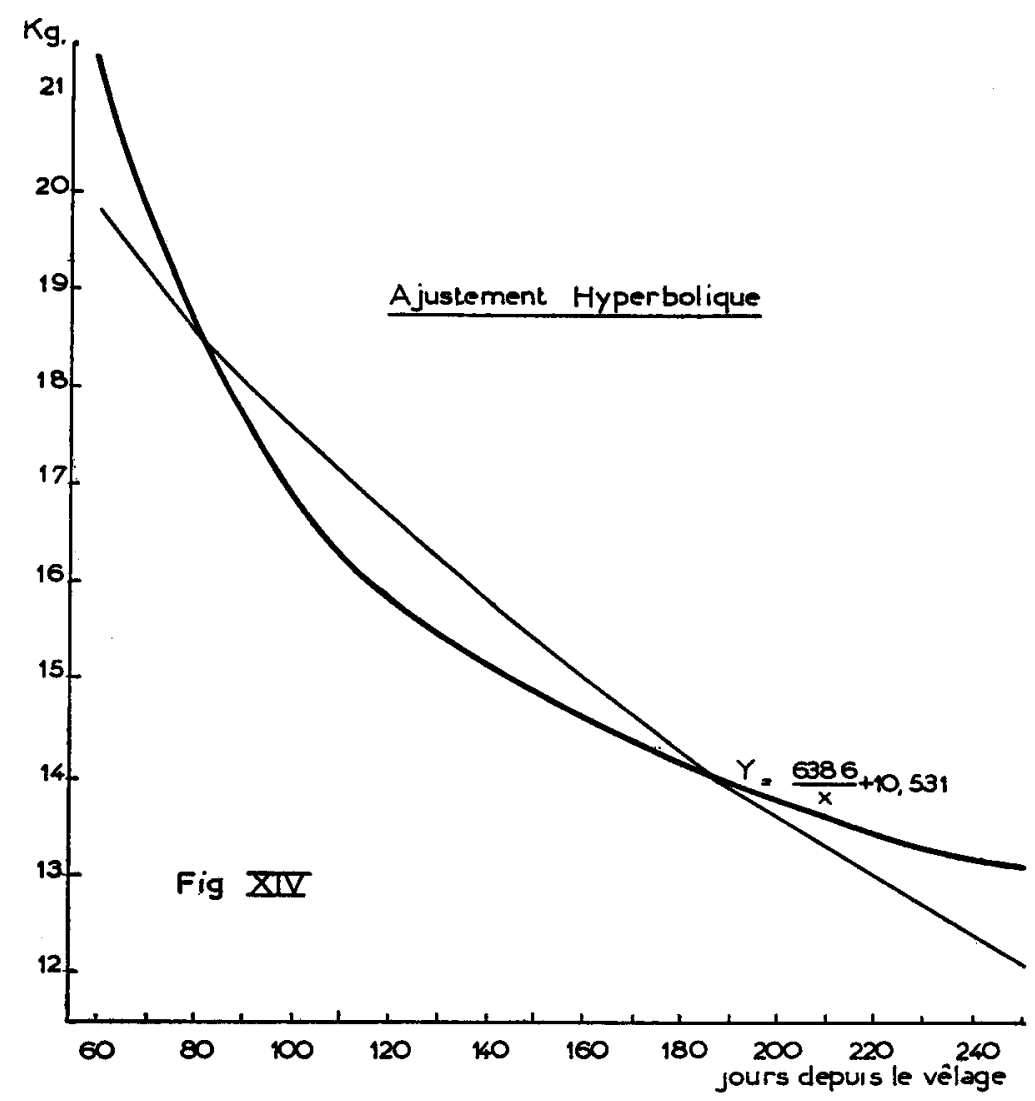

Le rapport de ces 2 variances est égal à $\frac{6 \mathrm{I}, 2}{24,6}=24,8$; au point $5 \%$ de la table de Snedecor, le rapport de ces deux variances indique que $s_{\mathrm{D}}^{2}$ est significativement plus grande que $s_{\mathbf{I}}^{2}$. 
I.'hypothèse d'une décroissance hyperbolique de la production laitière est donc infirmée.

\section{Hypothèse d'une régression parabolique}

Pour effectuer l'ajustement de nos données expérimentales à une régression parabolique d'équation $\mathrm{Y}=a x_{2}+b x+c$, oì $a, b, c$, sont des constantes à déterminer, nous avons opéré selon le même principe que précédemment.

Nous avons cherché à rendre minimum l'expression $\Sigma\left(\bar{y}_{i}-\mathrm{Y}_{i}\right)^{2}$ en annulant successivement les dérivées de cette expression par rapport à chacun des coefficients $a, b, c$.

Nous avons obtenu, alors, un système de 3 équations du I er degré en $a, b, c:$

$$
\left\{\begin{array}{c}
c=\bar{y}-b \bar{x}-\frac{a}{\mathbf{N}} \boldsymbol{\Sigma} x^{2} \\
b=\frac{\sum x y-c \mathrm{~N} \bar{x}-a \boldsymbol{\Sigma} x^{3}}{\boldsymbol{\Sigma} x^{2}} \\
a=\frac{\boldsymbol{\Sigma} x^{2} y-b \boldsymbol{\Sigma} x^{3}-c \boldsymbol{\Sigma} x^{2}}{\boldsymbol{\Sigma} x^{4}} .
\end{array}\right.
$$

Nous avons alors résolu ce système de 3 équations du I er degré à trois inconnues; nous avons tiré successivement les valeurs de $a, b, c$, en fonction de nos données expérimentales, et nous avons obtenu, pour nos 3 coefficients, les valeurs suivantes:

$$
\left\{\begin{array}{l}
a=97 \cdot 10^{-6} \\
b=-0,07 \\
c=23,64 .
\end{array}\right.
$$

L'équation de notre régression parabolique est donc :

$$
\mathrm{Y}=97 \cdot \mathrm{IO}^{-6} x^{2}-0,07 x+23,64
$$

dans laquelle $\mathrm{Y}$ représente la production théorique en $\mathrm{kg}$ et $x$ le temps en jours ; 23,64 représente la production fictive au vêlage si l'évolution de la production suivait une courbe parabolique depuis cette époque. (fig. I5).

\section{Valeurs statistique de l'ajustement parabolique}

La somme des carrés des écarts de la production moyenne observée à la production théorique d'ajustement $\Sigma\left(\bar{y}_{i}-Y_{i}\right)^{2}$ est très faible et égale à 0,034 , et justifie statistiquement 1'hypothèse d'une représentation parabolique. 
En prenant pour origine des temps $1 \mathrm{e} 6 \mathrm{o}^{\mathrm{e}}$ jour après le vêlage et en posant $\mathrm{X}=x-60$, l'équation de notre parabole devient :

$$
\mathrm{Y}=97 \cdot \mathrm{IO}^{-6} \mathrm{X}^{2}-0,058 \mathrm{X}+\mathrm{I} 9,8
$$

I9,8 qui représente la production théorique au $60^{\mathrm{e}}$ jour après le vêlage est, d'ailleurs, la production réelle.

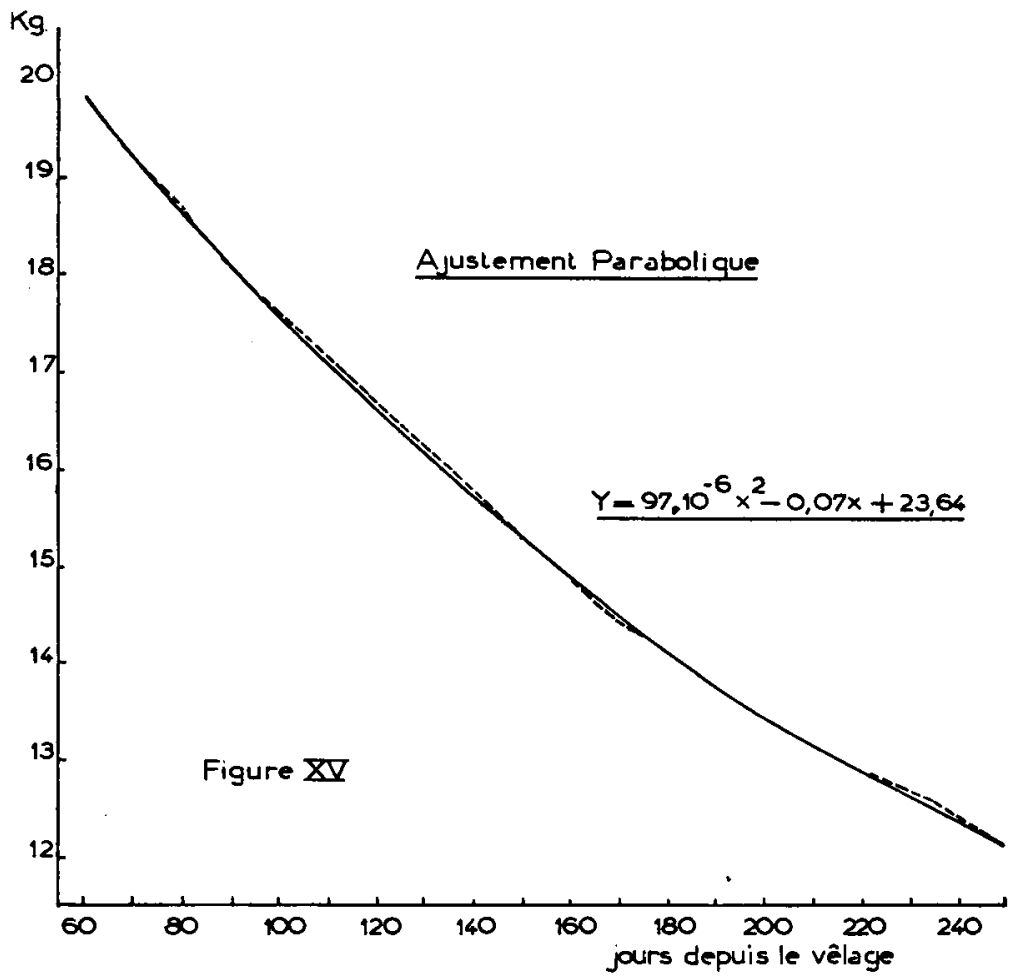

Il nous est possible, également, de compter le temps $T$ à partir de cette nouvelle origine en mois. Nous posons $T=\frac{X}{30} ;$ nous obtenons :

$$
\mathrm{Y}=873 \cdot \mathrm{IO}^{-4} \mathrm{~T}^{2}-\mathrm{I}, 74 \mathrm{~T}+\mathrm{Ig}, 8 .
$$

INTERPRÉTATION DE LA COURBE DE LACTATION ENTRE LE $60^{e}$ ET LE $25 \mathrm{O}^{\mathrm{e}}$ jOUR :

\section{Ligne de régression}

L'étude comparée de la valeur statistique des divers ajustements linéaire, exponentiel, hyperbolique et parabolique à la courbe moyenne de lactation, étudiée entre le $60^{\mathrm{e}}$ et le $25 \mathrm{o}^{\mathrm{e}}$ jour après le vêlage, montre que l'hypothèse d'une régression hyperbolique doit être seule rejetée.

L'examen de la somme des carrés des écarts des productions réelles aux productions théoriques d'ajustement figurant au tableau II, indique 
que la valeur de l'ajustement croît de la droite, à l'exponentielle, et à la parabole.

Dans ce dernier cas, en effet, cette somme $\left(\bar{y}_{i}-\mathrm{Y}_{i}\right)^{2}$ est respectivement I 3 fois et 49 fois inférieure aux données correspondantes pour les régressions exponentielle et linéaire.

Si l'ajustement de nos observations expérimentales à une droite, et surtout à une courbe exponentielle est statistiquement justifié, leur adaptation à une régression parabolique est parfaite et doit être préférée.

TABLEAU II

\begin{tabular}{|c|c|c|c|}
\hline Nature de l'ajustement & Équation théorique & $\Sigma\left(\bar{y}_{i}-Y_{i}\right)^{2}$ & $\begin{array}{l}\text { Valeur de } \\
\text { l'ajustement }\end{array}$ \\
\hline Hyperbole .. & $Y=\frac{638,6}{x}+10,53$ & $7,55^{6}$ & Mauvaise \\
\hline Droite........ & $Y=21,63-0,04 x$ & I,685 & Valable \\
\hline Exponentielle ......... & $\mathrm{Y}=22,77 \cdot 1 \mathrm{O}^{-0,00112 x}$ & 0,444 & Bonne \\
\hline Parabole..$\ldots \ldots \ldots \ldots$ & $Y=97 \cdot 10^{-6} x^{2}-0,07 x+23,64$ & 0,034 & Excellente \\
\hline
\end{tabular}

\section{Productions au $60^{\mathrm{e}}$ jour}

Le $60^{\mathrm{e}}$ jour marque le début de la phase de décroissance que nous avons étudiée; il peut être intéressant de comparer à la production moyenne, réellement observée, les productions théoriques que nous obtenons à cette même date, avec nos différentes hypothèses, statistiquement vérifiées.

Le tableau. III donne ces divers résultats :

Production au $6 \mathrm{o}^{\mathrm{e}}$ jour

\begin{tabular}{c|c|c|c}
\hline \hline Production observée & $\begin{array}{c}\text { Avec l'hypothèse } \\
\text { linéaire }\end{array}$ & $\begin{array}{c}\text { Avec l'hypotèse } \\
\text { exponentielle }\end{array}$ & $\begin{array}{c}\text { Avec l'hypothèse } \\
\text { parabolique }\end{array}$ \\
\cline { 1 - 2 } & 19,80 & 19,50 & 19,78 \\
\hline
\end{tabular}

I'hypothèse parabolique nous fournit la meilleure approximation de la production au $60^{\mathrm{e}}$ jour.

\section{Persistance}

Pour comparer la persistance de la lactation expérimentale aux persistances impliquées par les différentes hypothèses d'ajustement, nous avons choisi, comme élément de comparaison, le coefficient mensuel de persistance, mesuré par le rapport de la production d'un mois à celle du mois précédent. 
Le tableau IV compare aux coefficients expérimentaux les coefficients déduits des 3 hypothèses statistiquement justifiées. Pour établir ces divers coefficients, nous avons calculé les productions mensuelles moyennes à partir des 3 productions évaluées tous les ro jours, chaque mois.

TABLEAU IV

\begin{tabular}{|c|c|c|c|c|c|c|}
\hline Classes & $\begin{array}{l}\text { Mois de } \\
\text { lactation }\end{array}$ & $\begin{array}{l}\text { Productions } \\
\text { mensuelles }\end{array}$ & $\begin{array}{l}\text { C.P. (I) } \\
\text { observé }\end{array}$ & $\begin{array}{l}\text { Hypothèse } \\
\text { linéaire }\end{array}$ & $\begin{array}{l}\text { Hypothèse } \\
\text { exponentielle }\end{array}$ & $\begin{array}{l}\text { Hypothèse } \\
\text { parabolique }\end{array}$ \\
\hline $\begin{array}{l}70 \\
80 \\
90\end{array}$ & $3^{\text {ème }}$ & $P_{3}=18,627$ & C.P. $4 / 3=0,919$ & C.P. ${ }^{2} \cdot 4 / 3=0,934$ & C.P. $4 / 3=0,9^{26}$ & C.P. $4 / 3=0,917$ \\
\hline $\begin{array}{l}\text { I0O } \\
\text { I } 10 \\
\text { I } 20\end{array}$ & $4^{e m e}$ & $P_{4}=I 7, I I 9$ & C.P. $5 / 4=0,921$ & C.P. $5 / 4=0,930$ & C.P. $5 / 4=0,9^{26}$ & C.P. $5 / 4=0,919$ \\
\hline $\begin{array}{l}\text { I } 30 \\
\text { I } 40 \\
\text { I } 50\end{array}$ & $5^{\text {ème }}$ & $P_{5}=I_{5.777}$ & C.P. $6 / 5=0,918$ & C.P. $6 / 5=0,925$ & C.P. $6 / 5=0,926$ & C.P. $6 / 5=0,9^{2} 3$ \\
\hline $\begin{array}{l}\text { I } 60 \\
\text { I } 70 \\
\text { I } 80\end{array}$ & 6 ème & $P_{6}=I_{4}, 488$ & C.P. $7 / 6=0,93 \mathrm{I}$ & C.P. $7 / 6=0,919$ & C.P. $7 / 6=0.926$ & C.P. $7 / 6=0,929$ \\
\hline $\begin{array}{l}190 \\
200 \\
210\end{array}$ & $7^{\text {ème }}$ & $P_{7}=13,490$ & C.P. $8 / 7=0,939$ & C.P. $8 / 7=0,912$ & C.P. $8 / 7=0,926$ & C.P. $8 / 7=0,937$ \\
\hline $\begin{array}{l}220 \\
230 \\
240\end{array}$ & 8 ème & $P_{3}=12,674$ & & & & \\
\hline
\end{tabular}

( $\mathrm{c}$ C. P. $=$ coefficient mensuel de persistance.

L'hypothèse de la décroissance linéaire implique que la production du lait décroît mensuellement d'une quantité constante, égale à la pente de la droite, soit avec nos données I, $2 \mathrm{~kg}$. Dans ces conditions, le coefficient mensuel de persistance doit décroître du $60^{\mathrm{e}}$ au $25^{\circ} \mathrm{e}$ jour, ce qui ne s'accorde pas avec les données expérimentales.

L'hypothèse d'une décroissance exponentielle suppose que la production du lait diminue mensuellement, suivant un rapport constant. Le coefficient mensuel de persistance défini par ce rapport, doit être alors constant. I1 s'évalue dans notre étude à $0,926 \pm 0,003$.

Dans l'hypothèse de la décroissance parabolique que nous avons précédemment définie, le terme en $T$, dont le paramètre est négatif, traduit un processus de décroissance linéaire. Le terme en $\mathrm{T}^{2}$ dont le paramètre est positif, tend à freiner la diminution de la production et son action croît comme le carré du temps. Dans ces conditions, le coefficient mensuel de persistance doit augmenter, au cours de la période étudiée, et ce processus se vérifie avec les coefficients expérimentaux. Ceux-ci se classent sur le tableau IV en deux groupes, de part et d'autre du $6^{\mathrm{e}}$ mois ; avant cette époque, ils sont inférieurs au coefficient moyen ; ensuite, ils sont supérieurs. 
Cette variation, en accord avec l'hypothèse parabolique montre la nécessité d'étudier chaque coefficient mensuel de persistance et non de se limiter au seul coefficient moyen.

I a production laitière commence donc à la fin $\mathrm{du} 2^{\mathrm{e}}$ mois pour diminuer rapidement, puis subit, à partir du $6^{\mathrm{e}}$ mois, une décroissance plus lente, jusqu'au $250^{\mathrm{e}}$ jour.

Un moyen commode pour caractériser la lactation entre $1 \mathrm{e} 6 \mathrm{o}^{\mathrm{e}}$ et le $250^{\mathrm{e}}$ jour consiste à établir la moyenne des coefficients mensuels de persistance correspondant respectivement aux périodes comprises du $60^{\mathrm{e}}$ au $I 80^{\mathrm{e}}$ jour, d'une part, et du $\mathrm{I} 8 \mathrm{O}^{\mathrm{e}}$ au $25^{\mathrm{e}}$ jour, d'autre part.

Nous avons groupé ces résultats dans le tableau $\mathrm{V}$.

\section{TABLEAU V}

\begin{tabular}{|c|c|c|c|}
\hline Courbe & $\begin{array}{l}\text { Coefficient de persistance } \\
\text { moyen du } 60^{\mathrm{e}} \\
\text { au } 240^{\mathrm{e}} \text { jour } \\
\text { C.P. } 4 / 3+\mathrm{C} \text {. P. } 5 / 4 \\
+ \text { C. P. } 6 / 5+\text { C. P. } 7 / 6 \\
\quad+\text { C. P. } 8 / 7\end{array}$ & $\begin{array}{l}\text { Coefficient de persistance } \\
\text { moyen du } 60^{e} \\
\text { au r } 80^{\mathrm{e}} \text { jour } \\
\text { C. P. } 4 / 3 \text { C C. P. } 5 / 4 \\
+ \text { C. P. } 6 / 5\end{array}$ & $\begin{array}{c}\text { Coefficient də persistance } \\
\text { moyen du I } 80^{\mathrm{e}} \text { jour } \\
\text { au } 240^{\circ} \text { jour } \\
\text { C.P. } 7 / 6+\text { C. P. } 8 / 7\end{array}$ \\
\hline & 5 & 3 & 2 \\
\hline $\begin{array}{l}\text { Réelle........ } \\
\text { Parabolique ... } \\
\text { Exponentielle. } \\
\text { Droite ...... }\end{array}$ & $\begin{array}{l}0,9255 \\
0,925 \\
0,926 \\
0,924\end{array}$ & $\begin{array}{l}0,919 \\
0,920 \\
0,926 \\
0,929\end{array}$ & $\begin{array}{l}0,935 \\
0,933 \\
0,926 \\
0,915\end{array}$ \\
\hline
\end{tabular}

La courbe parabolique résume, par conséquent, au mieux 1'évolution des coefficients mensuels de persistance.

ETUDE DE LA COURBE DE LACTATION MOYENNE ENTRE LE $250^{\circ}$ jOUR ET LE $300^{\mathrm{e}}$ JOUR.

A partir du $250^{\mathrm{e}}$ jour, la courbe moyenne exprime une chute rapide des productions. Le tableau VI traduit ces résultats :

TABLEAU VI

\begin{tabular}{|c|c|c|c|}
\hline Classes & Productions observées & Moyennes mensuelles & Coefficient de persistance \\
\hline $\begin{array}{l}250 \\
260 \\
270 \\
280 \\
290 \\
300\end{array}$ & $\begin{array}{l}\text { I } 2, \text { I } 50 \\
\text { I I } 895 \\
\text { I I, } 599 \\
\text { II ,2 I } 4 \\
\text { I0,706 } \\
\text { I0, I } 48\end{array}$ & $\begin{array}{l}\text { I I }, 88 \text { I } \\
\text { I } 0,689\end{array}$ & $\frac{\mathrm{I} 0,689}{\mathrm{II}, 88 \mathrm{I}}=0,88$ \\
\hline
\end{tabular}

Le coefficient de persistance est, alors, de 0,88 . 
Le début de cette période survient environ I Io jours après la saillie, car les animaux que nous avons utilisés pour notre étude étaient saillis en moyenne I 40 jours après le vêlage.

\section{c) Influence de la gestation}

\section{LES TRAVAUX ANTÉRIEURS}

Ces résultats nous ont conduits à examiner l'influence de la gestation sur la production laitière ; un grand nombre d'auteurs a, d'ailleurs, étudié ce problème. Les premiers travaux détaillés sur cette question, sont ceux de RAGSDALE et coll. $(47,48)$, qui pensent qu'il y a deux manières d'étudier l'effet de la gestation : soit comparer deux groupes d'animaux qui ont été saillis à des dates différentes, soit établir des graphiques de production en gardant constante la période de lactation, mais en faisant varier la période de gestation. De leurs graphiques, établis pour le $8 \mathrm{e}$, le $9^{\mathrm{e}} \ldots \mathrm{I} 2^{\mathrm{e}}$ mois de lactation, les auteurs concluent qu'il y a une décroissance rapide de la production après le $5^{\mathrm{e}}$ mois de gestation. Une gestation à un stade précoce de la lactation peut entraîner une baisse totale de production de 220 à $300 \mathrm{~kg}$. Ces résultats sont confirmés, d'ailleurs, par les recherches de GowEN (49), qui chiffre l'effet dépressif de la gestation sur des vaches Guemesey entre I $80 \mathrm{~kg}$ et $270 \mathrm{~kg}$. L'auteur signale, en outre, qu'il y a des différences avec l'âge des animaux dans ces réductions de productions dues à les gestation. GaInes et Davidson (24) corrigent l'équation de décroissance normale de la production, par un terme soustractif, dont l'influence débute à la saillie, et dont l'action n'est vraiment sensible que vers $1 \mathrm{e} 5^{\mathrm{e}}$ mois de gestation. L'équation exponentielle de production $y=\mathrm{A} e^{-k t}$ devient, alors, $y=\mathrm{A} e^{-k t}-\mathrm{B} e^{k(t-c)}$, dans laquelle $B$ représente une nouvelle constante que les auteurs trouvent, d'ailleurs, proportionnelle à $\mathrm{A}$, où $\mathrm{K}$ est une constante qui figure le rythme de changement normal dans l'inhibition de la secrétion, due à la gestation, et $\mathrm{c}$ le nombre de mois du vêlage à la conception, $t-\mathrm{c}$, le nombre de mois depuis la saillie fécondante. GaINEs (25) corrige également l'équation des productions cumulées en faisant intervenir, comme variable des temps, l'intervalle de vêlage $x$, compté en mois. En admettant une durée de tarissement de 2 mois avant un nouveau vêlage, l'équation devient :

$$
\mathrm{Y}=\frac{a}{k}\left[\mathrm{I}-e^{-k ;(x-2)}\right] \text {. }
$$

Ainsi, est évident l'effet de la variation de l'intervalle de vêlage sur la production laitière.

Un grand nombre d'auteurs s'est attaché à mettre en relief l'époque à laquelle la gestation exerce une influence sensible sur la production. 
Gavin (8), Sanders (50), Pontecorvo (27), Ostergaard (ii), BünGER (34) aboutissent aux mêmes conclusions que GaINES et coll., RAGSDALE et coll. La décroissance de la production, selon eux, s'accélère au $5^{\mathrm{e}}$ mois de gestation; EDWARDs (I4) constate ce phénomène vers le II $2^{e}$ jour, et plus tard, Morrison et coll. (5I) entre le I $8^{\mathrm{e}}$ et le $\mathrm{I} 4 \mathrm{O}^{\mathrm{e}}$ jour.

LuDwick et coll. (43), dans la méthode qu'ils préconisent pour caractériser la persistance, indiquent que la production durant la $3^{\mathbf{e}}$ période de 8 o jours est légèrement influencée par la gestation, mais que, durant la $4^{\text {e }}$ et dernière période, 1'effet de la gestation est, par contre, très net. Si des coefficients différents sont affectés à chacun des rapports c'est que ces derniers n'interviennent pas de la même façon dans l'expression de la persistance moyenne.

Johansson et coll. (42) pensent, - que pour celles qui ne sont pas des premières lactations - la meilleure mesure de la persistance, est le rapport $\mathrm{P}_{2} / 1$ - que nous avons évoqué précédemment -, car il n'y a pas de corrections à apporter dans la longueur de l'intervalle de vêlage.

LörTsCHER (52) constate que dès la saillie, la décroissance de la production est plus marquée. LERoY et coll. (13) conseillent de diminuer de $2 \%$ le coefficient mensuel de persistance dès l'établissement de la gestation.

Hammond et SaNders (53) proposent des corrections pour les différentes longueurs de l'intervalle vêlage-saillie; (la durée de référence choisie pour cet intervalle est de roo jours). SANDERS (54) montre par ailleurs que des intervalles de vêlage de I2 à I3 mois sont à conseiller dans la pratique.

LIEVENS (55) rapporte en Belgique toutes les productions de contrôle laitier au cas normal d'une vache vêlant régulièrement tous les ans, vers la même époque, et commençant par conséquent, une nouvelle gestation. 3 mois après le vêlage. Pour chaque mois de retard de la saillie après le vêlage, la production en 300 jours est réduite de $5 \%$ avec une réduction maximum de $15 \%$. Pour une vache vêlant à nouveau dans l'année, la production obtenue en 300 jours est augmentée de $5 \%$ pour chaque mois en dessous de 12.

TuFf (56) a proposé, à la suite d'une étude des courbes de lactation de vaches norvégiennes, de prendre, comme unité de mesure de la lactation, la production durant les I 80 premiers jours. En effet, à cette date, les variations entre lactations, dues aux variations dans la longueur de l'intervalle de vêlage, ne sont pas marquées et ne nécessitent par conséquent, aucune correction. ZorN et coll. (57) puis DinkHauser $\left(5^{8}\right)$ ont confirmé, par la suite, en Allemagne, les résultats de Tuff. L'expression de la persistance de Mahadevan (44) correspond, d'ailleurs, à la même idée (59).

Enfin, une étude récente d'ERB et coll. (60) apporte de réelles pré- 
cisions sur l'effet de la gestation sur la production laitière. Selon ces auteurs, le rythme de décroissance dans la production est 3 ou 4 fois plus rapide $d u I 8 I^{e}$ au $223^{e}$ jour de gestation que du $I 0 I^{e}$ au $I 80^{e}$ jour. L'inhibition de la sécrétion laitière se produit approximativement au même moment quels que soient le nombre de traites journalières, 1'âge des animaux - ce qui est contraire aux résultats de GowE, - la race, le mois de saillie, le niveau de production de la vache, ou l'intervalle de vêlage tant qu'il ne dépasse pas 18 mois.

\section{NOS RÉSUL,TATS :}

Notre étude nous a montré qu'environ 4 mois après la saillie, il y avait une chute plus rapide de la production, particulièrement accusée à partir du $\mathrm{I} 3 \mathrm{O}^{\mathrm{e}}$ jour de gestation.

Nous avons observé, d'autre part, deux lots de 8 vaches respectivement saillies 80 jours et $I 45$ jours après le vêlage. Une chute assez nette de production s'enregistre dans les deux cas environ 3 mois après la saillie, soit au $190^{\mathrm{e}}$ jour pour le premier lot et au $26 \mathrm{o}^{\mathrm{e}}$ jour pour le $2^{\mathrm{e}}$ lot. La figure $\mathbf{I} 6$ représente les courbes moyennes de production correspondantes.

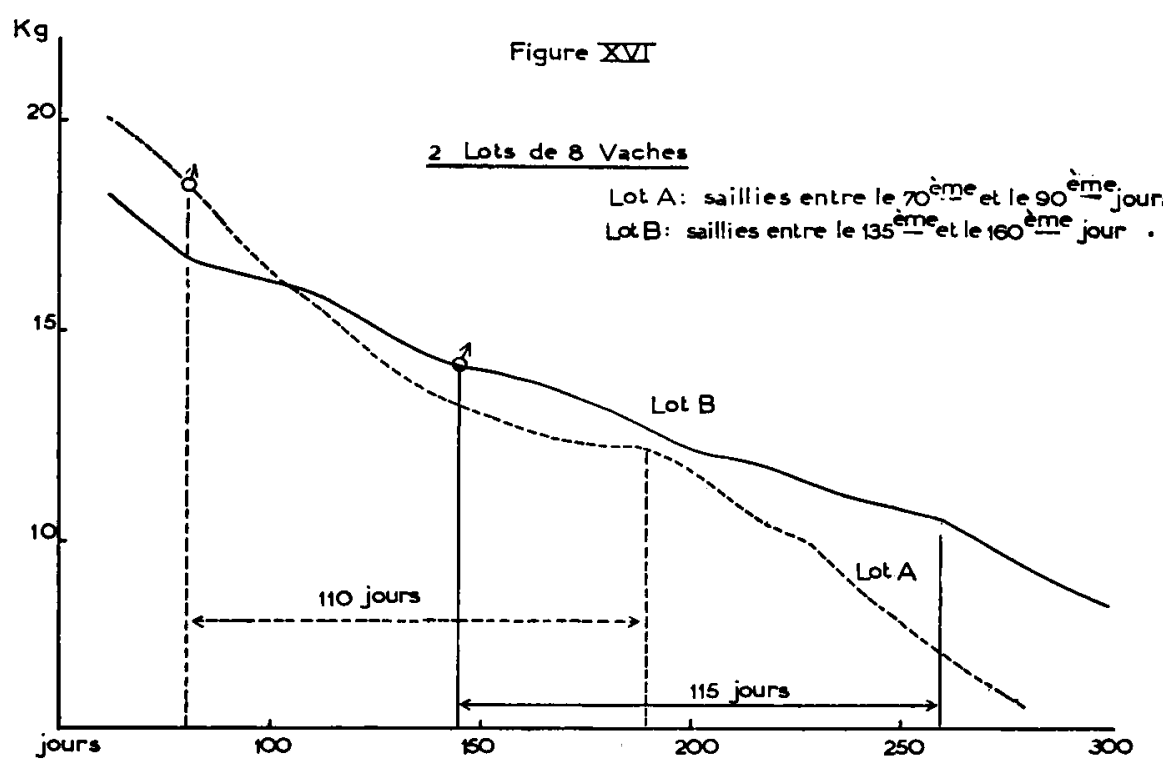

Par ailleurs, la représentation parabolique de la courbe de lactation entre le $60^{\mathrm{e}}$ et le $25^{\mathrm{e}}$ jour après le vêlage nous conduirait à penser que la gestation, dans la première phase, durant les 4 premiers mois, n'a pas, comme l'ont affirmé GAINEs et coll., I,EROY et co 11., LörTSCHER, une influence néfaste sur la production laitière. Il semble, au contraire que la persistance de la lactation s'accroisse pendant cette période. L'évolution des coefficients mensuels de persistance est assez suggestive à cet égard. 
Ainsi, au cours d'une période qui s'étend du $60^{\mathrm{e}}$ au $\mathrm{I} 8 \mathrm{o}^{\mathrm{e}}$ jour après le vêlage, la production laitière diminue selon un rythme de décroissance relativement rapide, sans que le coefficient mensuel de persistance soit inférieur à $0,9 \mathrm{I} 5$. Du $\mathrm{I} 8 \mathrm{O}^{\mathrm{e}}$ au $25 \mathrm{O}^{\mathrm{e}}$ jour, la production décroît suivant un rythme légèrement plus lent; le coefficient mensuel de persistance augmente progressivement; sa valeur moyenne est 0,935. Ia gestation exerce ensuite, durant une deuxième phase, une influence inhibitrice caractérisée sur la production laitière; au cours de cette période, nous avons $v u$ que le coefficient mensuel de persistance tombait à 0,88 .

Beaucoup d'interprétations physiologiques ont été données à l'effet inhibiteur de la gestation sur la production. Nous ne les envisagerons pas en détail ici. On peut penser, cependant, qu'une sécrétion accrue d'œstrogènes au bout d'un certain temps de gestation, inhibe la lactation. Des études sont en cours pour tenter d'expliquer dans leur ensemble, nos résultats.

\section{d) Conclusions sur l'étude de la courbe moyenne}

Deux lois de régression, l'une de forme exponentielle, l'autre de forme parabolique peuvent être retenues pour exprimer la phase de décroissance de la lactation. Si la première est d'une utilisation plus pratique, notamment à des fins de sélection, il est indiscutable, cependant que

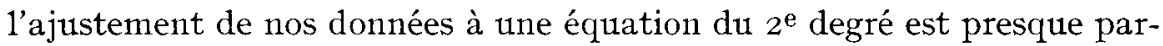
faite, et qu'il nous a révélé deux phases dans l'effet de la gestation sur la lactation.

I a gestation entraîne en effet une baisse de production environ 4 mois après la saillie, baisse précédée d'un phénomène tampon qui accroît légèrement la persistance.

Il apparaît alors nécessaire de tenir compte de l'intervalle vêlagesaillie — " service period "des Anglos-Saxons — comme $7^{\mathrm{e}}$ paramètre nécessaire pour définir une lactation $d s$ (voir fig. I).

La persistance doit être en définitive estimée dans un intervalle de temps compris entre la date de la production maximum et la fin des 4 premiers mois de gestation. Dans la pratique, les vaches sont rarement saillies dans les deux mois qui suivent le vêlage ; ainsi la production durant les 6 premiers mois ne serait pratiquement jamais perturbée par l'effet de la gestation, des comparaisons sont alors possibles entre les persistances des divers animaux.

En fait, chez une vache, la lactation s'intègre dans un cycle régulier de reproduction ; il faut done la définir et l'étudier en fonction de ce cycle. Nous empruntons à un rapport du Milk Marketing Board (6I) une courbe moyenne de production correspondant à un important échantillon 
d'animaux contrôlés (fig. I7). Les différents paramètres de position de la lactation y sont indiqués, dans le cadre de l'intervalle de vêlage, unité de temps en matière de reproduction.

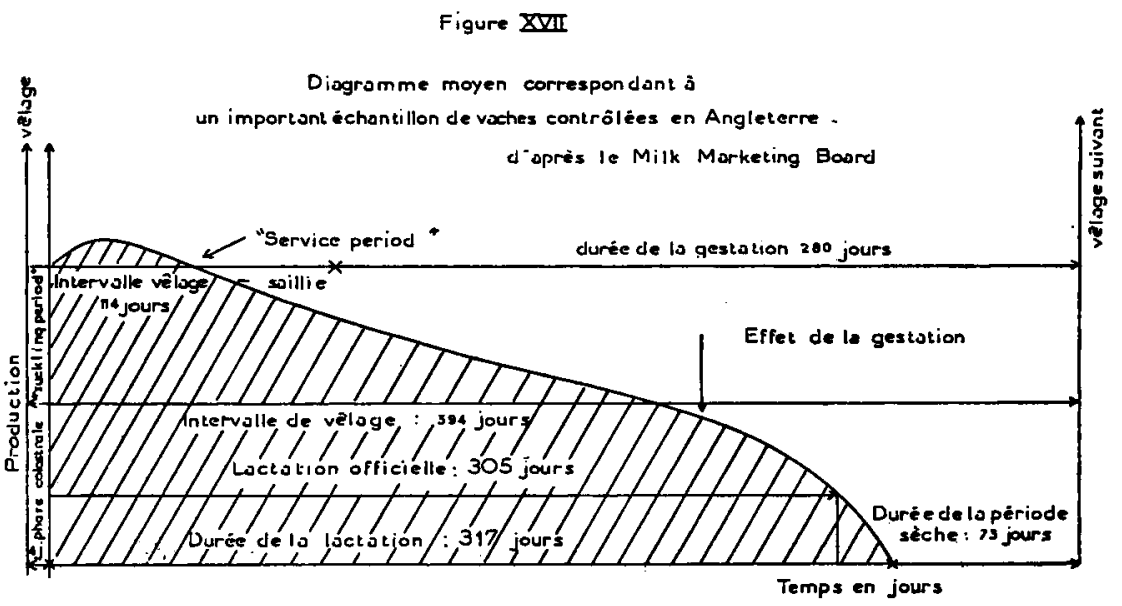

Signalons en outre que Johansson et Hansson (42) ont étudié très en détail les diverses corrélations existant entre une lactation caractérisée par une certaine quantité de lait ou de matières grasses et sa place dans la vie de l'animal ( $\mathrm{n}^{\circ}$ d'ordre de la lactation, âge de l'animal quand elle a débuté) ; sa place vis-à-vis des variations saisonnières (mois de vêlage) ; divers paramètres de l'intervalle de vêlage qui l'ont précédée; divers paramètres enfin de l'intervalle de vêlage dans laquelle elle se situe.

\section{III. - CONCLUSION}

Il nous a semblé utile de chercher à définir dans les meilleures conditions la forme de la courbe de lactation.

Pour des études de nutrition et le contrôle de certains facteurs physiologiques agissant sur la production laitière, il est intéressant pour un chercheur, de savoir à quelle régression adapter les productions observées de ses animaux, au cours d'une longue période expérimentale. Avec l'emploi de la méthode des périodes successives, on peut en effet calculer à partir de 2 phases pré-et post-expérimentales, la production théorique qui aurait dû être enregistrée pendant la phase expérimentale, pour la comparer à la production réelle de l'animal durant cette période. Si des études de courte durée sont effectuées, le calcul d'une régression linéaire est suffisant.

L'excellent ajustement de nos données avec une régression parabolique nous a permis de serrer de près l'effet de la gestation sur la production laitière. Il nous paraît d'ailleurs souhaitable de tenir compte des 
résultats précédents dans la tenue des fiches de contrôle laitier, en notant très exactement la date de la saillie fécondante et en calculant les coefficients mensuels de persistance dans les conditions que nous avons précisées plus haut.

La courbe de lactation est d'autre part un excellent test biologique des conditions dans lesquelles les vaches laitières ont été entretenues et elle fournit des renseignements précieux sur la valeur de l'affourragement distribué dans une étable. Ainsi est-il possible, à l'examen des graphiques de contrôle laitier, de déceler dans un troupeau des périodes de sousalimentation. Lors de la délivrance des certificats de lactation on attire d'ailleurs l'attention des éleveurs sur les chutes anormales de production. Ragsdale et TURNER (62) signalaient déjà en I923 qu'en cas de sous-alimentation la production laitière était réduite, cette réduction dépendant de la longueur de la période de sous-nutrition et de l'avancement de la lactation. Johansson et Hansson (42) affirment également que la sous-nutrition affecte, et la production maximum, et la persistance. (Par contre, il semble démontré que la suralimentation n'a aucun effet sur la persistance, TURNeR (63); BONNIER (I8)).

La courbe de lactation a, par surcroît, un intérêt économique. La productivité d'une vache laitière dépend en effet de trois facteurs :

- la fréquence de ses vêlages,

- son niveau de production maximum,

- sa persistance.

Il n'est pas économiquement indifférent qu'une production déterminée soit obtenue en un temps donné, au cours d'une lactation avec un maximum élevé et une faible persistance ou inversement, avec un maximum plus faible et une persistance plus forte.

En effet, il peut être intéressant pour l'éleveur de répartir sa production d'une façon assez uniforme sur l'ensemble de l'année (prix différents du lait d'hiver et d'été).

D'autre part, des vaches avec de fortes productions initiales sont plus difficiles à alimenter rationnellement à cause de leur forte pointe de production. Des déséquilibres alimentaires peuvent se produire au cours de cette période dont les répercussions sont sensibles, non seulement sur la lactation en cours, mais sur les lactations ultérieures, la fertilité de l'animal, et en définitive son âge de réforme.

En outre, les besoins en aliments concentrés non produits sur 1'exploitation sont plus importants pour des vaches qui ont atteint des maxima de production élevés, comme le signalent ENGELER (64) et ESKEDAL (2). Ces constatations font ressortir tout l'intérêt zootechnique d'animaux à courbes de production régulières.

Enfin, il est nécessaire de chercher à connaître les effets respectifs de l'hérédité et du milieu avec ses multiples composantes (âge, mois de 
vêlage, niveau de nutrition...) sur la persistance, et les rapports de cette persistance avec le maximum de production. De nombreux et importants travaux ont été réalisés à ce sujet par Gaines, Sanders (65), Pontecorvo (27), Johansson et Hansson (42), Ludwick et coll. (66), Sikka (28), Mahadevan (I4). Afin de préciser si la persistance est une caractéristique individuelle pouvant être améliorée par sélection, il est indispensable de calculer la corrélation existant entre les valeurs des persistances des lactations d'une même vache, et l'héritabilité de cette persistance. (CsuKAS (67) pense que la moyenne de 3 lactations d'un même animal donne une bien meilleure estimation de sa courbe de production). Les résultats de Johansson (68), Sikka (28), Mahadevan (44) semblent indiquer que ce caractère n'est que faiblement héritable. Nous nous proposons, dans une étude ultérieure, de réexaminer cet important problème.

\section{IV. - RÉSUME}

La courbe moyenne de production de $\mathbf{I} 46$ vaches de race française Frisonne Pie Noire, traites 2 fois par jour, a été analysée statistiquement dans sa phase de décroissance; $96 \%$ des animaux avaient dépassé leur maximum de production au $60^{\mathrm{e}}$ jour.

Deux périodes ont été distinguées dans la partie descendante de la lactation, l'une du $60^{\mathrm{e}}$ au $250^{\mathrm{e}}$ jour, l'autre du $250^{\mathrm{e}}$ au $300^{\mathrm{e}}$ jour après le vêlage. Diverses hypothèses de régression ont été testées et ont permis d'affirmer qu'une fonction parabolique représentait fort bien la décroissance des productions au cours de la première période.

La gestation, 4 mois après son début, a un effet dépressif caractérisé sur la lactation. Par contre, pendant ces 4 premiers mois, et conformément à l'hypothèse d'une régression parabolique, la persistance de la lactation aurait tendance à s'accroître, fait qui semble important à souligner. Des études sont en cours pour essayer d'expliquer ces résultats.

Les auteurs discutent les différentes expressions de la persistance, et pensent que cette persistance doit être étudiée entre la période de la production maximum et l'époque où l'effet de la gestation est sensible, c'est-à-dire environ 4 mois après la saillie fécondante. La moyenne arithmétique des coefficients mensuels de persistance leur paraît un moyen pratique pour caractériser cette persistance, à des fins de sélection, ou pour le contrôle des conditions d'alimentation dans une étable.

\section{BIBLIOGRAPHIE}

(I) Gaines (W. L.), Davidson (F. A.). - Relation between percentage fat content and yield of milk; correction of milk yield for fat content, Illinois Agr. Exp. Sta. Bull., 245, p. 577, I923. 
(2) Eskedal (H. W.). - The ideal dairy cow ; shape of lactation curve ; an important economical question. Communications du Ve Congrès International de Zootechnie, Paris, p. 29, I949.

(3) Eskedal (H. W.). - Communication aux Journées d'Étude de la F. E. Z., Utrecht, I95I.

(4) HaEcker (A. I.). - Experiments with dairy herd, Nebraska Agr. Exp. Sta. Bull., 76, p. 2I, I903.

(5) Turner (C. W.), Ragsdalf (A. C.), Brody (S.). - How the advance of the period of lactation affects the milk flow? J. Dairy Sc., 6, p. 527, I923.

(6) MCCANDlish (A. C.). - The influence of the stage of lactation on the production of dairy cows, $J$. Dairy Sc., \%, p. 255, I924.

(7) DRAKELEy (T. J.), White (M. K.). - The influence of the stage of lactation and the breed of the cow on the yield and quality of the milk, $J$. Agr. Sc., 1\%, p. II9, I927.

(8) Gavin (W.). - Studies on milk records; the influence of foetal growth on yield, J. Agri. Sc., 5, p. 309, I9I3.

(9) BARTLETT (S.). - Studies in milk secretion based on the variations and yields of milk and butterfat produced at morning and evening milkings, J. Agri. Sc., 19, p. 36, I929.

(IO) OXLEY (C. D.). - A study of progress of lactation in relation to the milk yield and the butterfat percentage of milk produced by cows of Shorthorn type, $J$. Dairy Res., 6, p. II3, I935.

(II) Ostergand (P. S.). — Pat réf. Le Lait, 14, p. 735, I934. Recherches statistiques sur la production de lait de quelques vaches danoises inscrites au H. B., Copenhague, I93I.

(I2) MöllgaARD (H.). - Grundzünge der Ernährungsphysiologie der Haustiere, éd. Parey, Berlin, p. 3I0, I93I.

(13) LeRoy (A. M.), Sentex (J.), STOECKEL (R.). - Le producteur de lait, éd. Hachette, Paris, p. 95, 1946.

(I4) EDWARDS (J.). - - Factors influencing the relationship between the secretion of milk and butterfat, $J . A g r . S c ., 40$, p. Ioo, I950.

(I5) Csukas (Z.). - The temporair performance in milk yield as a basis for selection in dairy cattle. Communications du Ve Congrès International de Zootechnie, Paris, p. I3, I949.

(I6) SturTEVANT (A. H.). - Influence of distance from calving on milk yield, New-york Agr. Exp. Sta. An. Rep., 5, p. 26, I 886 (par réf. suivante).

(I7) Garnes (W. L.). - Measures of persistency of lactation, J. Agr. Res., 34, p. 373, 1927.

(I8) BONNIER (G.). - Is the shape of the lactation curve genetically determined ? Hereditas, 20, p. I99, I935.

(I9) Kronacher (C.), von Patow (C.), von Frings (P.). — Einiges über Milchleistungen in Dahlemer Rassen und Forschungsstall, Ztschr. fiir Tierziuchtung, 36, p. II9, I936.

(20) Brody (S.), Ragsdal (A. C.), Turner (C. W.). - The rate of the decline of milk production with the advance of the period of lactation, J. Gen. Phys., 5, p. 44I, I923.

(2I) Brody (S.), TURner (C. W.), Ragsdale (A. C.). - The relation between the initial rise and subsequent decline of milk secretion following parturition, J. Gen. Phys., 6, p. 54I, I924.

(22) Gannes (W. I.). - Interpretation of the lactation curve, $J$. Gen. Phys., 9, p. 325, I926.

(23) Gaines (W. L.). - Persistency of lactation in dairy cows, Illinois $A g r$. Exp. Sta. Bull., 288, p. 355, I927.

(24) Gaines (W. L.), Davidson (F. A.). - The effect of advance in lactation and gestation on mammary activity, J. Gen. Phys., 9, p. 325, r926.

(25) Gaines (W. L.). - Milk yield in relation to recurrence of conception, J. Dairy Sc., 10, p. II7, 1927. 
(26) Gooch (M.). - An analysis of the time change in milk production in individual lactations, $J$. $A g r$. Sc., 25, p. 7I, I935.

(27) Ponteconvo (G.). - A study of the persistency in a herd of Ayrshire cows, J. Dairy Res., 11, p. II3, I940.

(28) Sikka (L. C.). - A study of the lactation as affected by heredity and environment, J. Dairy Res., 1\%, p. 23I, I950.

(29) IINFIELD (F. B.). - Experiments with dairy cows. - A study of the records of the dairy herd for 5 years, Utah Sta. Bull., 68, p. I67, rgoo.

(3o) Grady (R. I.). - Stage of lactation affects milk yield, Mo. Bull. Ohio Agr. Exp. Sta., 12, p. 4OI, I9I7.

(3I) TuRner (C. W.). - A quantitative form of expressing persistency of milk or fat secretion, J. Dairy Sc., 9, p. 203, I926.

(32) Leroy (A. M.). - Nouvelle méthode pour le contrôle de l'alimentation des vaches laitières, $C . R$. Acad. A gri., 28, p. 937, I93I.

(33) Leroy (A. M.). - Méthode de contrôle de l'alimentation des vaches laitières, Le Lait, 14, p. 366, I934.

(34) BÜNGER (H.). - Kann man die Milchleistungen im Verlauf einer Laktation mit einiger Zuverlässigkeit im voraus bestimmen und kann man aus dem Verlauf der Laktation zuverlässige Schlïsse auf die Richtigkeit der Fiitterung ziehen ? Ztschr. für Tierzüchtung, 58, p. 58, I949.

(35) Sanders (H. G.). - The shape of lactation curve, J. Agri. Sc., 13, p. I69, Ig23.

(36) Sanders (H. G.). - The analysis of the lactation curve into maximum yield and persistency, $J$. $A g r$. Sc., 20, p. I45, I930.

(37) Fredericksen (I.), OstergaARd (P. S.). - Stambogskoers Moelkeydelse, IXe Congrès International de Laiterie ; Copenhague, I93I (par référence, Nielsen).

(38) Carryle (W. L.), Woli (F. W.). - Studies in milk production, Wisconsin, A gr. Exp. Sta. Bull., 102, p. 88, I903.

(39) BRUUN (E.). - L Lypsykauden maidontuotantokäyrään vaikuttavista tekijoïstä, Helsinki, p. I2I, I 928 (pour référence Johansson et Hansson).

(40) TERHo (T.). - Undersökningar över inhemska tjurars inverkan pä avkommans mjölkproduction och fetthalt i mjölken. Stat. Lantbruksförs Vetensk., Helsinki, 4, p. I57 ; I 937 (pour référence, Johansson et Hansson).

(4I) Johansson (I.). - Undersökninga röver avkastningsresultaten inom thorsätrabesättningen under Stallfodring och betesgäng, Lantbruksakademien Handl. och Tidskr., \%6, p. 77I, I937 (pour référence la suivante).

(42) Johansson (I.), Hansson (A.). - Causes of variation in milk and butterfat yield of dairy cows, Kungl. Lantbruksadademiens Tidskrift, p. 64, I940.

(43) LUDWICK (T. M.), PETERSEN (W. E.). - A measure of persistency of lactation in dairy cattle, $J$. Dairy Sc., 26, p. 439, I943.

(44) Mahadevan (P.). - The effect of environment and heredity on lactation. II Persistency of lactation, J. Agric. Sc., 41, p. 89, I95I.

(45) Niessen (K.). - Uber die Form der Laktationskurve, Ztschr. fiur Tierzüchtung, 39, p. 9, I937.

(46) BARTLETT (S.). - Normal day to day variability of yield of milk and fat of individuals cows, J. Agri. Sc., 19, p. 438, I 929 .

(47) Ragsdale (A. C.), Turner (C. W.), Brody (S.). - The effect of gestation upon lactation in the dairy cows, J. Dairy Sc., \%, p. 24, I924.

(48) Brody (S.), Ragsdale (A. C.), Turner (C. W.). - The effect of gestation on the rate of decline on milk secretion with the advance of the period of lactation, J. Gen. Phys., 5, p. 777, I923.

(49) Gowen (J. W.). - Intrauterine development of the bovine fetus in relation to the milk yield in Guernesey cattle, J. Dairy Sc., r, p. 3II, I924.

(50) Sanders (H. G.). - The variations in milk yield caused by season of the year, service, age and dry period and their elimination II Service, J. Agri. Sci., 1\%, p. 503, 1927. 
(5I) Morrison (R. A.), Erb (R. E.), Shaw (A. O.). - Effect of estrus and gestation upon milk yield and butterfat content and the normal daily variation in fat test of Holstein cows, Proc. W. Div. Amer. Dairy Sc. Ass., p. 5I, I950.

(52) LÖRTSCHER (H.). - Variationsstatistiche Untersuchungen an Leistungserhebungen in einer British-Friesian Herde, Ztschr. für Tierzüchtung, 39, p. 268, I937.

(53) Hammond (J.), SANDERS (H. G.). - Some factors affecting milk yield, J. Agri. Sc., 13, p. 74, I923.

(54) Sanders (H. G.). - The length of the interval between calvings, J. Agri. Sc., 17, p. 2I, I927.

(55) LIEVENs $(\vec{F}$.$) . - Considérations sur le contrôle laitier en Belgique, Revue$ de l'A griculture, 1, p. 9, I95I.

(56) TuFF (P.). - Faktoren die auf die Milchleistungen wirken, Ztschr. für Tierzïchtung, 29, p. I42, I932.

(57) ZORN (W.), FUNKE (A.). - Die Milchleistungen in den ersten I8o Tagen der Laktation beim Rind und ihre Bedetitung als Masstab fïr die Milchleistungsfähigkeit und deren erbliche Veranlagung in der Rinderzucht, Ziichtungskunde, 10, p. I93, I939.

(58) Drnkhauser (F.). - Die frühzeitige Errkundung der Leistungsfähigkeit und des Erbwertes in Milchmenge und Fettgehalt beim Rind auf Grund der Leistung in den ersten I8o Melktagen, Mitteilungen für die Landw., 59, p. 727, I944.

(59) Mahadevan (P.). - The effect of environment and heredity on lactation. I-Milk yield, $J . A g r . S c ., 41$, p. 8o, I95I.

(60) Eirb (R. E.), Goodwin (M. M.), Morrison (R. A.), Shaw (A. O.). Lactation studies. I. Effect of gestation, J. Dairy Sc., 35, p. 224, I952.

(6I) Milk Marketing Board. - Report of the production division, 1, p. 24, 9950.

(62) RAgSdale (A. C.), TURNER (C. W.). - The effects of underfeeding on milk secretion, J. Dairy Sc., 6, p. 25I, I923.

(63) Turner (C. W.). - A study of the relation between feed consumption and milk secretion, J. Dairy Sc., 7, p. 535, r924.

(64) ENGELER (W.). - Le type et le standard du bétail dans les conditions de l'économie agricole suisse. Rapports particuliers du Ve Congrès International de Zootechnie, Paris, p. 28I, I949.

(65) SANDERS (H. G.). - The variations in milk yields caused by season of the year, service, age and dry period and their elimination. I. Season of year; III. Age; IV. Dry period and standardisation of yields, J. Agri. Sc., 17, p. 339, I927, 18, p. 46, p. 209 , I928.

(66) Ludwick (T. M.), Pettersen (W. E.), FitCh (J. B.). - Genetic aspects of persistency in dairy cattle, J. Dairy Sc., 26, p. 448, I943.

(67) Csukas (Z.). - The genetics of the lactation curve. Proc. $7^{\text {th }}$ International Genetics Congress; Edinburgh, p. 99. I939.

(68) Johansson (I.). - Production traits in dairy cattle and their improvment by selection. Rapports particuliers du Ve Congrès International de Zootechnie, Paris, p. 278, I949.

\section{Ouvrages de statistique}

LHÉRITIER (P.). - Les méthodes statistiques dans l'expérimentation biologique ; C. N. R. S., Paris, I949.

VESSEREAU (A.). - Méthodes statistiques en biologie et en agronomie; éd. Baillère, Paris, I948. 